ARTICLE

DOI: $10.1038 / s 41467-018-05099-3$

\title{
Lgl1 controls NG2 endocytic pathway to regulate oligodendrocyte differentiation and asymmetric cell division and gliomagenesis
}

\author{
Mathieu Daynac ${ }^{1}$, Malek Chouchane ${ }^{1}$, Hannah Y. Collins ${ }^{1,8}$, Nicole E. Murphy ${ }^{1}$, Noemi Andor (1) 1,9, Jianqin Niu², \\ Stephen P.J. Fancy 2,3 , William B. Stallcup ${ }^{4}$ \& Claudia K. Petritsch (1) 1,5,6,7
}

Oligodendrocyte progenitor cells (OPC) undergo asymmetric cell division (ACD) to generate one OPC and one differentiating oligodendrocyte $(\mathrm{OL})$ progeny. Loss of pro-mitotic proteoglycan and OPC marker NG2 in the OL progeny is the earliest immunophenotypic change of unknown mechanism that indicates differentiation commitment. Here, we report that expression of the mouse homolog of Drosophila tumor suppressor Lethal giant larvae 1 ( $\operatorname{Lg} \mid 1$ ) is induced during OL differentiation. $L g / 1$ conditional knockout OPC progeny retain NG2 and show reduced $\mathrm{OL}$ differentiation, while undergoing more symmetric self-renewing divisions at the expense of asymmetric divisions. Moreover, Lg/1 and hemizygous Ink4a/Arf knockouts in OPC synergistically induce gliomagenesis. Time lapse and total internal reflection microscopy reveals a critical role for Lgl1 in NG2 endocytic routing and links aberrant NG2 recycling to failed differentiation. These data establish Lgl1 as a suppressor of gliomagenesis and positive regulator of asymmetric division and differentiation in the healthy and demyelinated murine brain.

\footnotetext{
${ }^{1}$ Department of Neurological Surgery, University of California San Francisco, San Francisco, CA 94143, USA. ${ }^{2}$ Department of Pediatrics, University of California San Francisco, San Francisco, CA 94158, USA. ${ }^{3}$ Department of Neurology, University of California San Francisco, San Francisco, CA 94158, USA. ${ }^{4}$ Sanford Burnham Prebys Medical Discovery Institute, San Diego CA 92037, USA. ${ }^{5}$ Brain Tumor Center, University of California San Francisco, San Francisco, CA 94158, USA. ${ }^{6}$ Helen Diller Comprehensive Cancer Center, University of California San Francisco, San Francisco, CA 94158, USA. ${ }^{7}$ Eli and Edythe Broad Center of Regeneration Medicine and Stem Cell Research, University of California San Francisco, San Francisco, CA 94143, USA. ${ }^{8}$ Present address: Department of Neurobiology, Stanford University, Stanford, CA 94305, USA. ${ }^{9}$ Present address: Department of Medicine, Stanford University, Stanford, CA 94305, USA. These authors contributed equally: Mathieu Daynac, Malek Chouchane. Correspondence and requests for materials should be addressed to C.K.P. (email: claudia.petritsch@ucsf.edu)
} 
A dult oligodendrocyte progenitor cells (OPC) expressing the proteoglycan NG2 (CSPG4) continuously divide and generate differentiating oligodendrocytes $(\mathrm{OL})$ throughout adulthood ${ }^{1}$. OPC divisions are frequently asymmetric and generate progeny of distinct fate, where only one of the daughter cells preserves NG2 expression and the opposite daughter cell downregulates $\mathrm{NG}^{2,3}$. Thus far, asymmetric distribution of NG2 protein is the earliest immunophenotypic change amongst daughter cell pairs generated by asymmetric cell division (ACD). NG2-positive daughter cells proliferate at higher rates than NG2negative cells, showing that the early phenotypic asymmetry correlates with distinct short-term fate ${ }^{2}$. NG2 contributes to establishing this cell fate bias within newly generated cell pairs after ACD. NG2 binds platelet-derived growth factor-AA (PDGFAA) and PDGF receptor alpha (PDGFR $\alpha$ ) and thereby enhances PDGFRa signaling and promotes timely OPC proliferation ${ }^{4-7}$. Moreover, long-term fate tracking of the ACD progeny showed that early NG2 asymmetry permanently affects cell fate. The NG2-positive progeny of ACD retain OPC characteristics while the NG2-negative progeny upregulate $\mathrm{CC} 1$, a marker for commitment to the OL fate, and eventually differentiate ${ }^{2,8,9}$. Importantly, chemical-induced demyelination increases the rates of NG2 asymmetry 9 . Collectively, the data underline that asymmetric distribution of NG2 marks and also actively generates different OPC progeny. Furthermore, ACD balances OPC proliferation with differentiation in the normal brain and generates $\mathrm{OL}$ in demyelinated lesions to contribute to remyelination ${ }^{2,3,9,10}$. It is unclear how the downregulation of NG2 protein is achieved in the differentiating oligodendrocyte. This lack of mechanistic insights into ACD and early differentiation limits our understanding of brain homeostasis.

OPC give rise to glioma in genetically engineered mouse models ${ }^{11-14}$. When undergoing neoplastic transformation, OPC show higher rates of symmetric self-renewing divisions at the expense of $\mathrm{ACD}^{2}$. These data suggest that downregulation of NG2 in OPC progeny is critical for ACD, differentiation, and attenuation of tumorigenesis ${ }^{2}$.

Surface levels of the membrane-spanning NG2 protein are regulated by clathrin-mediated endocytosis in mouse embryonic fibroblasts ${ }^{15}$. Clathrin-mediated or receptor-mediated endocytosis is a multi-step process, whereby membrane-localized proteins are engulfed as cargo in clathrin-coated pits, which then bud off the membrane to form the early endosome. Cargo proteins are then sorted into either the recycling endosome and re-integrated into the membrane or targeted to the late endosome and subsequently the lysosome for degradation ${ }^{16}$. It is not known whether NG2 trafficking by the endocytic pathway is important for NG2 downregulation and OL differentiation.

The WD40 repeat-containing protein lethal giant larvae (Lgl) was initially characterized as a tumor suppressor gene, in Drosophila melanogaster ${ }^{17}$, and it was subsequently shown to mediate ACD of Drosophila neuroblasts ${ }^{18}$. Lgl is an evolutionary conserved protein that initiates cell polarity by recruiting proteins to membrane subdomains (for review, see ref. ${ }^{19}$ ). As one of two mammalian $L g l$ genes $^{20}$, Lgll is highly expressed in the brain ${ }^{21}$. Lgll knockout studies during mouse embryogenesis have revealed a function for Lgl1 in polarity and adherens junction integrity in neuroepithelial cells ${ }^{22}$, and in suppressing proliferation of dorsal telencephalon radial glial progenitors at early postnatal stages ${ }^{23}$. A bona-fide tumor suppressor role for Lgll in gliomagenesis is supported by studies showing that loss of tumor suppressor $P T E N$, a frequent and early driver mutation in glioblastoma ${ }^{24}$, inhibits Lgll activity ${ }^{25}$. Constitutively active Lgll inhibits invasion and induces differentiation of primary human glioblastoma cells $^{26}$. Nevertheless, a tumor suppressive role for Lgll in OPC has not been investigated yet.
Here, we report that $L g l 1$ expression is upregulated during OL differentiation and that Lgll protein is detected in committed OL in the adult murine brain. Lgll conditional knockout (cKO) OPC show a differentiation defect characterized by an aberrant coexpression of NG2 with OL commitment markers. Moreover, in Lgll cKO OPC rates of ACD are reduced while rates of symmetric, self-renewing divisions and proliferation are increased, in both intact and chemically demyelinated corpus callosum (CC). Lgll knockout synergizes with hemizygous Ink4a/Arf knockout in OPC to induce gliomagenesis. Time lapse imaging of surfacelabeled, endocytosed NG2 shows decreased co-localization with the lysosome in Lgll cKO OPC. Additionally, total internal reflection microscopy reveals that in Lgl1 cKO OPC NG2 bypasses degradation and is rather recycled to the membrane. Aberrant NG2 recycling is linked to defective OPC differentiation. Thus, we establish Lgl1 as a positive regulator of differentiation and ACD and suppressor of gliomagenesis. We suggest that Lgl1 promotes differentiation by supporting NG2 routing to the lysosome.

\section{Results}

Lgl1 expression is upregulated in OL differentiation. To unravel the mechanism for NG2 down-regulation, we searched the RNA-Seq transcriptome database ${ }^{27}$ for conserved ACD regulator genes with altered expression in OL versus OPC. We noticed a four-fold increase of Lgl1 (Llgl1) transcript in newly formed OL versus OPC, suggesting that Llgl1 expression is increased upon OL differentiation. To directly test this notion, OPC were isolated from the white matter area of the CC of postnatal days 30-60 (P30-P60) mice and cultured in parallel under proliferation and differentiation conditions (Fig. 1a). We performed immunofluorescence staining for OPC marker NG2 and mature OL marker O4 to show differentiation. As expected, we found O4-expressing cells displaying a branched OL morphology only under differentiation but not under proliferation conditions (Fig. 1b). We used quantitative real-time PCR (qrtPCR) to assess changes in Llgl1 transcript levels with differentiation and aging. Analyses reveal a significant increase in Llgll transcript under differentiation conditions $(2.5$-fold, $p<0.01)$ (Fig. 1c), along with differentiation markers Mbp, Slc1a1, Ndrg1, GPR17, and Mog. We noted that OPC markers, such as Cspg4 (NG2), Olig2, and Sox10 are unchanged. Llgl1 transcript levels increase with age, being higher at P60 in OPC, and reaching a peak at P180 in OL (Supplementary Figs. 1d, e).

To test whether Lgll protein expression is detectable in differentiated OL in situ, as suggested by our transcript analyses, we co-immunostained P35 wildtype mouse brain sections for Lgl1, OPC markers PDGFRa, and NG2 (Fig. 1d, Supplementary Fig. 1a), and committed OL marker $\mathrm{CCl}$ (Fig. 1e) ${ }^{8}$. A quantification of all detectable Lgl1-positive cells in the CC revealed that more Lgl1-expressing cells are CC1 positive (59\%) than PDGFRa (14\%) and NG2 (22\%) positive, respectively (Fig. 1f, g). Expression of Lgl1 is undetectable in NG2-positive OPC in the subventricular zone (Supplementary Figs. 1b, c).

Hence, Lgll expression increases with differentiation and overlaps largely with differentiating OL, suggesting therefore its potential role in a differentiation mechanism.

Lgl1 knockout in OPC blocks OL differentiation. The expression pattern suggests that Lgll positively regulates OL differentiation. In order to investigate a putative role for Lgll in OPC differentiation, we used a mouse model where Lgll knockout is targeted to NG2 cells ${ }^{28}$. We bred NG2-Cre ${ }^{E R T 2}$ driver mice ${ }^{9}$ with red-fluorescence protein Cre-reporter (Ai14-Tom ${ }^{29}$ and Lgl1 floxed $\left(L g l 1^{f l f l}\right)^{21,23}$ mice, as well as Lgl1 wildtype $\left(L g l 1^{w t / w t}\right)$ mice 
a

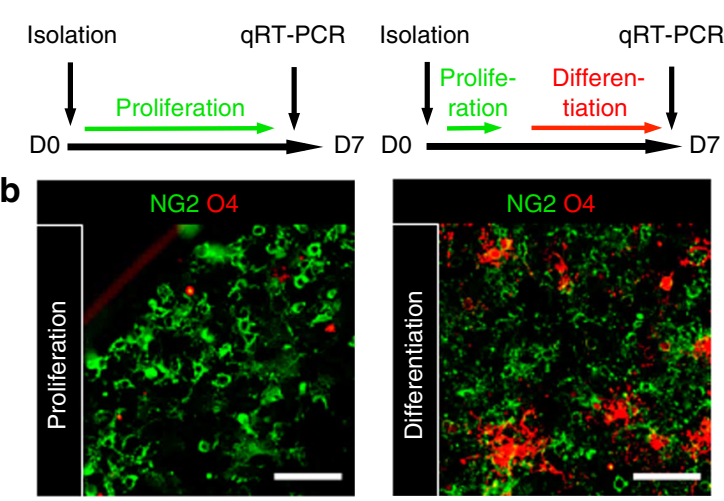

d

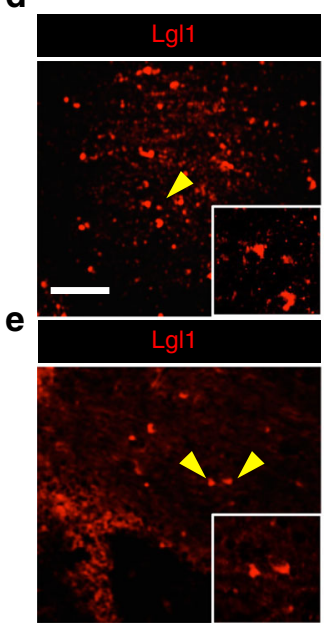

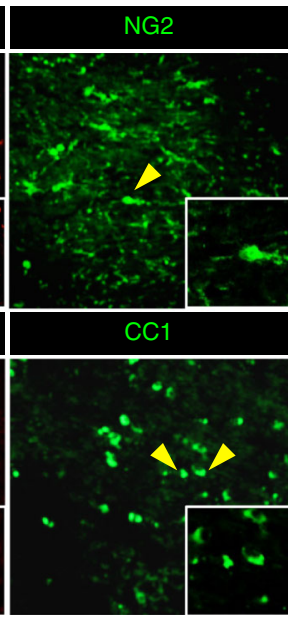

C

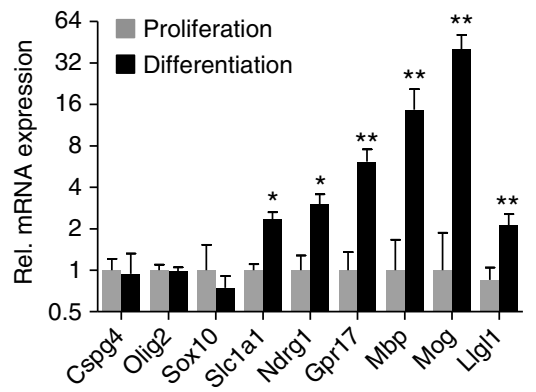

Fig. $1 \mathrm{Lg} / 1$ is expressed in oligodendrocyte progenitor cells (OPC) and its expression increases with differentiation into oligodendrocytes. a Scheme of OPC treatment before qRT-PCR. b Immunofluorescence for OPC marker NG2 and oligodendrocyte differentiation marker O4 of corpus callosum-derived OPC under proliferating or differentiating conditions. Differentiation increases O4-positive cell frequency. Scale bar = $100 \mu \mathrm{m}$. c Graph representing mRNA expression level changes with differentiation. QRT-PCR results are displayed on a $\log _{2}$ scale and normalized to proliferative conditions. Data represented as mean \pm s.e.m. of five independent experiments $\left(n=5,{ }^{\star} p<0.05,{ }^{\star \star} p<0.01\right.$, Mann-Whitney test). d, e Co-immunostaining of P35 wildtype mouse corpus callosum for Lgl1 and OPC marker NG2 (top panels) or differentiation marker CC1 (bottom panels). Insets are high magnification panels of Lgl1 positive cells (yellow arrows). Scale bar: $100 \mu \mathrm{m}$. f Schematic of coronal section of a P60 mouse showing the corpus callosum where cells were counted. $\mathbf{g}$ Quantification of PDGFR $\alpha$-positive, NG2-positive, and CC1-positive cells per total Lg11-positive cells. 14\% were PDGFR $\alpha$-positive, 22\% were NG2-positive, and 59\% were CC1-positive. Data are represented as mean \pm s.e.m. of four independent experiments

as controls. Compound transgenic mice were injected with tamoxifen once at P30 and recombined cells were identified by immunofluorescence staining for red fluorescent protein dTomato (aka Tom + cells) (Fig. 2a). NG2 and CC1 co-staining (Fig. 2b) revealed that amongst Tom + cells, NG2 + CC1 + cells were increased (WT $=14.7 \%$ vs. $\mathrm{cKO}=30.3 \% ; p<0.01)$, $\mathrm{CC} 1+$ cells were decreased $(\mathrm{WT}=51.8 \%$ vs. $\mathrm{cKO}=37.6 \% ; p<0.01$ ) and NG2+ cells were not significantly changed in Lgl1 cKO vs. Lgl1 WT CC (Fig. 2c). These data show that Lgl1 is not required for CC1 expression in OPC progeny. Lgl1 is rather required for NG2 protein down-regulation and positively regulates OL differentiation.

To provide further evidence that Lgl1 positively regulates OL differentiation, we analyzed ex vivo isolated Lgli cKO cells (Fig. 2d) for expression of OL maturation marker O4 and OPC marker PDGFRa at P60 (Figs. 2e, f, Supplementary Figs. 2a, b) and at P120 (Supplementary Figs. 2c, d). Indeed, we find that the total number of OPC increased to a greater extent than OL $(\mathrm{OPC}=4.5$ fold $\mathrm{cKO}$ vs. WT; $p=0.002 ; \mathrm{OL}=2.3$ fold $\mathrm{cKO}$ vs. WT; $p=0.06$ ) at P60 (Fig. 2e). Accordingly, the frequency of
OPC amongst Tom + , recombined cells increased in $L g l 1 \mathrm{cKO}$, while the frequency of OL decreased amongst Tom + cells $(\mathrm{OPC} / \mathrm{WT}=62 \% \quad$ vs. $\mathrm{OPC} / \mathrm{cKO}=79 \% ; \quad \mathrm{OL} / \mathrm{WT}=33 \% \quad$ vs. $\mathrm{OL} / \mathrm{cKO}=19 \%$; Fig. $2 \mathrm{f}$ ). At P120, the frequency of OPC was still higher amongst $L g l 1 \mathrm{cKO}$ Tom + cells than amongst Lgll WT Tom + cells $(\mathrm{OPC} / \mathrm{cKO}=41.3 \%$ vs. $\mathrm{OPC} / \mathrm{WT}=23.4 \% ; p=0.02)$ and the frequency of $\mathrm{OL}$ was still lower $(\mathrm{cKO}=39.4 \%$ vs. $\mathrm{WT}=51.7 \% ; p=0.02$; Supplementary Fig. $2 \mathrm{~d}$ ).

Taken together, these data show that upon Lgl1 knockout, OL aberrantly retain NG2 protein expression and expand as OPC marker positive cells, while failing to further differentiate and mature.

In contrast to postnatal and adult OPC, embryonic OPC are not committed to the oligodendrocyte lineage and reportedly also generate astrocytes or neurons $9,30,31$. To test if Lgll deletion affects the lineage commitment in young adult OPC, we quantified expression of neuroblast and astrocyte markers CD24 and GLAST, respectively, by flow cytometry (Supplementary Figs. 2a, b). As expected, only a small fraction of recombined cells expressed neuroblast and astrocyte markers $(<2 \%)$ and the 
a

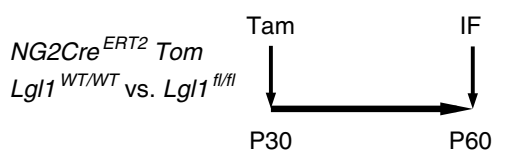

b

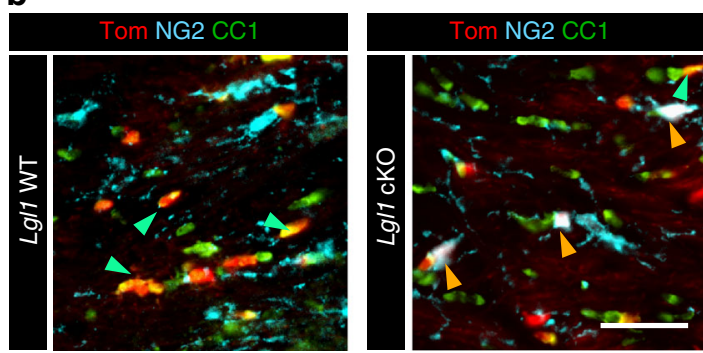

d

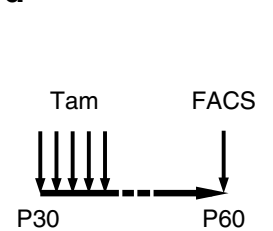

e

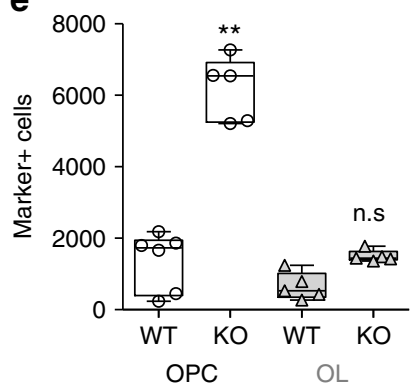

C

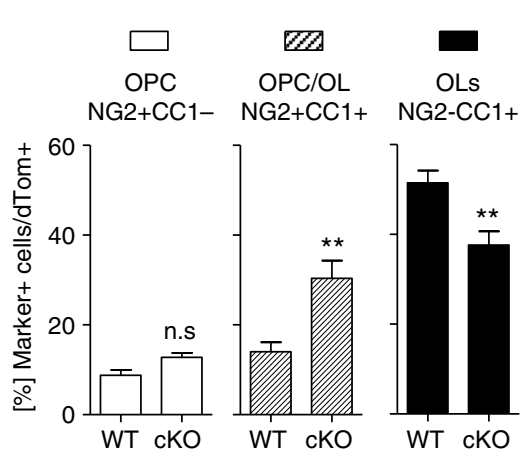

f
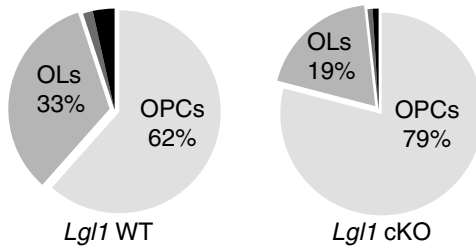

h

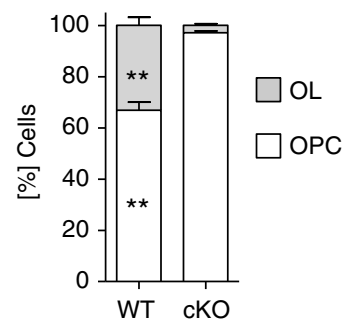

Fig. 2 Lgl1 knockout blocks OPC to OL differentiation. a Schematic of approach for clonal labeling and NG2 Cre-conditional deletion of Lg/1 in OPC. b Representative triple immunostaining of corpus callosum of $L g / 1$ WT and $L g / 1 \mathrm{cKO}$ from mice treated as in (a) for red fluorescent protein (Tom), OPC marker NG2 and differentiation marker CC1. Green arrows point to Tom+CC1+ oligodendrocytes. Orange arrows in the $\mathrm{Lg} / 1 \mathrm{cKO}$ panel point to Tom+NG2 $+\mathrm{CC} 1+$ cells. Scale bar: $50 \mu \mathrm{m}$. c Quantification of NG2+, CC1+, and NG2+CC1+ cells ratios amongst Tom+ cells. Data are represented as mean \pm s.e.m. from the analyses of $4 \mathrm{Lg} \mid 1$ WT vs. $4 \mathrm{Lgl1}$ cKO treated mice ( ${ }^{\star \star} p<0.01$, Mann-Whitney test). d Schematic of broad deletion of Lgl1 in P30 mice followed by FACS at P60. e Plot showing quantification of OPC (white) and OL (gray) in mice treated following scheme in (d). Data are represented as box \& whiskers \pm s.e.m. from the analyses of $6 \mathrm{Lg} \mid 1$ WT vs. $5 \mathrm{Lg} / 1 \mathrm{cKO}$ treated mice ( ${ }^{* *} p<0.01$, Mann-Whitney test). f Pie charts of quantification for NG2+, O4+, astrocyte marker GLAST+ and neuroblast marker CD24+ cells amongst Tom + cells, after Lgl1 knockout at P60 (see also Supplementary Fig. 2 b). Data are represented as percentages from the analyses of $6 \mathrm{Lg} / 1 \mathrm{WT}$ vs. $5 \mathrm{Lg} / 1 \mathrm{cKO}$ mice (OPC $p<0.01 ;$ OL $p<0.01$ ). $g$ Representative immunostaining for NG2 and $\mathrm{O} 4$ of OPC and OL isolated from GFAP-Cre, Tom, Lgl/wt/wt and, GFAP-CreERT2, Tom, Lg/7l/fl mice. Scale bars: $100 \mu \mathrm{m}$. h Quantification of NG2 and O4 cells amongst all red fluorescent protein (Tom) positive cells show that $L g / 1$ knockout strongly blocks the differentiation of OPC into O4+OL. Data are represented as mean \pm s.e.m. from the independent analyses of 4 Lgl1 WT vs. 4 Lgl1 cKO mice $\left({ }^{\star \star} p<0.01\right.$, Mann-Whitney test)

frequency of neuroblasts and astrocytes did not change significantly following Lgl1 deletion (Supplementary Fig. 2b). We concluded that Lgl1 loss does not alter the potential of young adult OPC to generate astrocytes and neuroblasts, suggesting a specific role of Lgl1 in OL differentiation.

Demyelinating injury increases the rate of OL proliferation and differentiation when compared with non-lesioned murine brain $^{32,33}$. To test if Lgl1 is also required for OL differentiation in demyelinating lesions, we quantified the frequency of mature OL marker Plp in lysolecithin-induced lesions in NG2-Cre ${ }^{E R T 2}$ Ail4-TdTom Lgl fllfl and $L g l 1^{w t / w t}$ mice injected with tamoxifen at P52 (Supplementary Figs. 2e, f). The frequency of Plp-positive cells amongst recombined cells was significantly decreased upon
Lgll deletion $(\mathrm{WT}=73.3 \%$ vs. $\mathrm{cKO}=33.0 \%$; $p<0.01$; Supplementary Fig. $2 \mathrm{~g}$ ), showing that Lgl1 is critical for OL differentiation in demyelinating lesions.

Lastly, we tested if the requirement for Lgl1 in differentiation can be recapitulated ex vivo. To this end, we isolated OPC from the corpus callosum of GFAP-Cre Lgll cKO and GFAP-Cre Lgll WT control mice and cultured them under OL differentiation conditions in vitro ${ }^{34}$ and stained cells for NG2 and O4. Quantifications revealed a significant increase of NG2+ cells and a decrease of $\mathrm{O} 4+$ cells amongst all Tom + cells, upon Lgl1 knockout (Figs. 2g, h).

Taken together, we conclude that Lgl1 is required for the downregulation of NG2 and OL differentiation. 
Lgl1 is required for asymmetric OPC divisions. Postnatal and adult OPC divide asymmetrically to generate at a one-to-one ratio an $\mathrm{NG} 2+\mathrm{OPC}$ and a CC1 $+\mathrm{OL}^{2,3,10}$. Given that $L g l 1$ knockout reduces the number of $\mathrm{CC} 1+$ cells (Fig. 2c), we hypothesize that Lgl1 regulates ACD. To test this hypothesis, we visualized and quantified ACD in Lgl1 NG2-Cre ERT2 Ai14-TdTom Lglifl/fl and $L g l 1^{w t / w t}$ mice that were injected with tamoxifen once at postnatal day 30 (P30) for clonal recombination ${ }^{35}$. To monitor proliferation, mice were treated with EdU for 14 days (Fig. 3a), and cell pairs were identified by co-immunostaining for Tom, $\mathrm{CC} 1$, and $\mathrm{EdU}^{2,3}$ (Fig. 3b). Cell pairs in the corpus callosum were scored as (i) symmetric, self-renewing (both cells = CC1 negative), (ii) asymmetric (one CC1-positive and one CC1-negative cell) and as (iii) symmetric differentiating (both cells = CC1 positive; Fig. 3b). The average cell cycle length of OPC increases from $~ 3$ days in P20 to 9 days in P60 animals ${ }^{1}$. Assuming a linear increase in cell cycle lengths between P30 and P45, recombined OPC should have divided two to three times during this period. Indeed, we were able to detect recombined cell pairs at $\mathrm{P} 45$. Amongst these, ACD rates were reduced in the $L g l 1 \mathrm{cKO}$ $(\mathrm{WT}=23.2 \%$ vs. $\mathrm{cKO}=12.5 \% ; p=0.023)$. Vice versa, symmetric, self-renewing divisions were increased in the Lgll cKO $(\mathrm{WT}=28.4 \%$ vs. $\mathrm{cKO}=51.2 \% ; p=0.028)$. Noteworthy, symmetric differentiated $(\mathrm{CC} 1+)$ cell pair rates were not significantly changed upon $L g l 1 \mathrm{cKO}(\mathrm{WT}=48.4 \%$ vs. $\mathrm{cKO}=36 \% ; p=0.2$ ) (Fig. $3 \mathrm{c}$ and Supplementary Table 1).

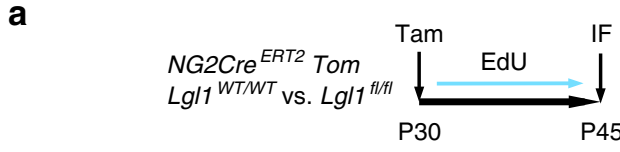

b
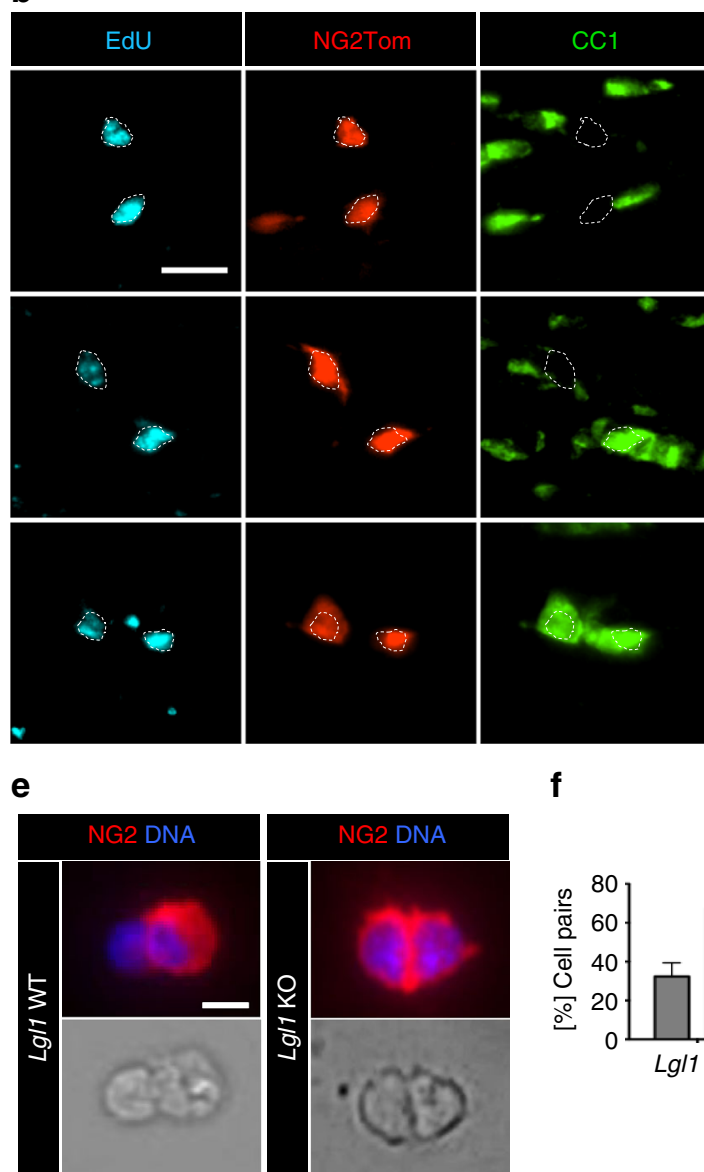

C

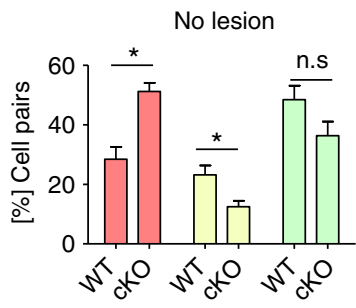

d division (ACD)

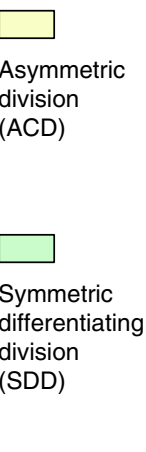

Symmetric differentiating division (SDD) self-renewing division (SSD)

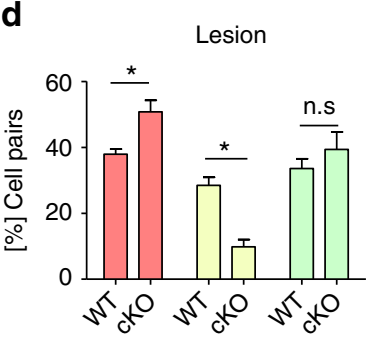

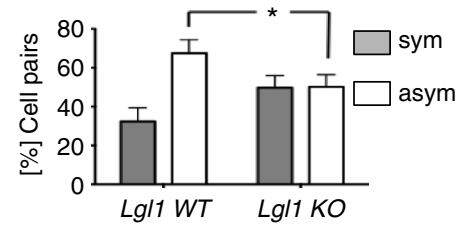

Fig. 3 Lgl1 regulates asymmetric cell divisions of OPC. a Treatment schematic for in vivo pair assay in P3O NG2-CreERT2 Tom Lgl1 WT vs. Lg/1 cKO mice, which were given EdU for 14 days in drinking water. b Left panels: Representative cell pairs in the corpus callosum of treated mice and co-immunostained for red fluorescent protein (Tom) to label NG2+ cells and progeny, proliferation marker EdU and CC1. Symmetric, self-renewing divisions = two Tom+EdU $+\mathrm{CC} 1-$ progeny, asymmetric cell division $=$ one Tom + EdU $+\mathrm{CC} 1-$ and one Tom + EdU $+\mathrm{CC} 1+$ progeny and symmetric, differentiating division $=$ two Tom + EdU $+\mathrm{CC} 1+$ progeny. Scale bar: $10 \mu \mathrm{m}$. c Graph depicting the frequency of each cell division mode and showing a significant decrease of asymmetric divisions (yellow bars) and an increase of symmetric, self-renewing divisions (red bars) in Lgll knockout (cKO) mice when compared with wildtype (WT) mice. Data are represented as mean \pm s.e.m. from the analyses of 4 Lgll WT vs. 4 Lgll cKO treated mice ( $n=4$, ${ }^{\star} p<0.05$, Mann-Whitney test). d Graph depicting the percentage of three division modes during remyelination in Lg/7 WT and Lg/1 cKO corpus callosum, and showing a significant decrease of asymmetric divisions (yellow bars) and increase of symmetric, self-renewing divisions (red bars). Data are represented as mean \pm s.e.m. from the analyses of 4 Lgll WT vs. 4 Lgll cKO treated mice ( $n=4,{ }^{\star} p<0.05$, Mann-Whitney test). e Representative NG2+ OPC pairs stained for NG2 (red) and DNA (blue) showing an asymmetric division in Lg/1 WT (left panel) and symmetric, self-renewing division in $\mathrm{Lg} / 1 \mathrm{cKO}$ (right panel) cells. Scale bar $=10 \mu \mathrm{M}$. $\mathbf{f}$ Graph depicting percentage of cell pairs and showing that Lgl1 loss increases symmetric, self-renewing divisions at the expense of asymmetric divisions in vitro $\left(n=6,{ }^{\star} p<0.05\right.$, Mann-Whitney test) 
Similarly, cell pairs in lysolecithin-induced demyelinating lesions in the corpus callosum and spinal cord, showed increased rates of symmetric self-renewing divisions at the expense of ACD upon Lgl1 knockout (Fig. 3d and Supplementary Figs. 3a, b and Supplementary Table 1).

To test for a disruption of ACD ex vivo, we generated Lgll KO and Lgl1 WT control OPC and plated them for pair assays, as described (Fig. 3e) ${ }^{2}$. We found that the frequency of ACD was reduced in Lgl1 $\mathrm{KO}$ OPC (WT $=72 \%$ vs. $\mathrm{cKO}=49 \%$ ) (Fig. 3f; $p=0.05$ ).

These data show that Lgl1 does not prevent CC1 expression, but rather prevents downregulation of OPC marker NG2, thereby lowering rates of asymmetric distribution of NG2. Thus, Lgl1 is required for ACD and restricts symmetric, selfrenewing divisions in the healthy and injured brain and in the injured spinal cord.
Lgl1 knockout increases OPC proliferation and promotes gliomagenesis. Given that Lgll knockout cells fail to restrict expression of pro-mitotic NG2, we hypothesize that their proliferation rates might be increased. We therefore assessed proliferation in NG2-Cre $e^{E R T 2}$ Ai14-TdTom Lgl1 $1^{f l f l}$ and Lgl1 ${ }^{\text {wt } / w t}$ mice injected with tamoxifen at P30 and exposed to EdU at P45 (Supplementary Figs. 4a-c) and at P60 (Figs. 4a, b and Supplementary Figs. 4d-f). Quantifications revealed that the overall percentage of Tom + cells at P60 was significantly increased in the Lgll cKO (14.8\%) vs. the Lgl1 WT corpus callosum $(7.9 \% ; p=0.0005$; Fig. 4a, b). Moreover, we chemically labeled EdU-positive $(\mathrm{Edu}+)$ cells. Edu + cells were 2.3-fold more frequent amongst the Tom+ cells in Lgll cKO vs. the Lgl1 WT corpus callosum ( $p=0.014$; Supplementary Figs. 4b, c) at P45. A similar increase was observed in demyelinated corpus callosum of P55 mice (1.8-fold higher EdU + cells amongst Tom + Lgl1 cKO vs. Lgl1 WT; $p=0.007)$

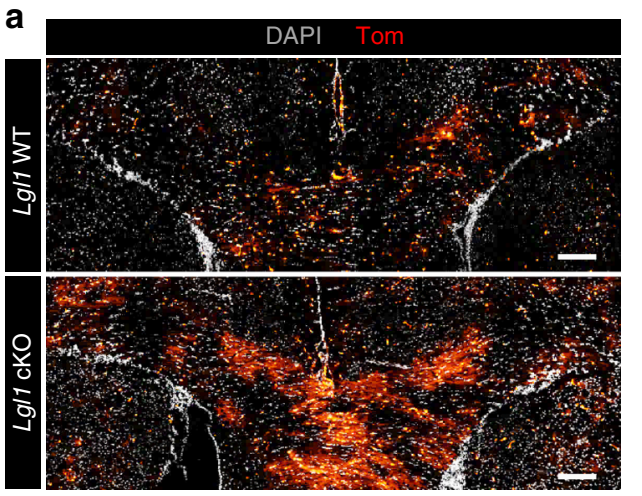

\section{b}

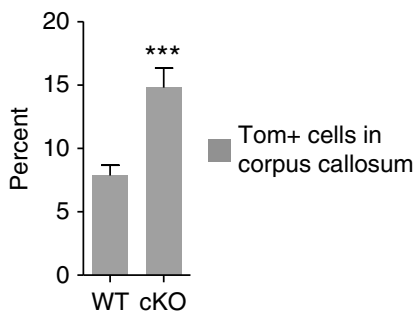

C

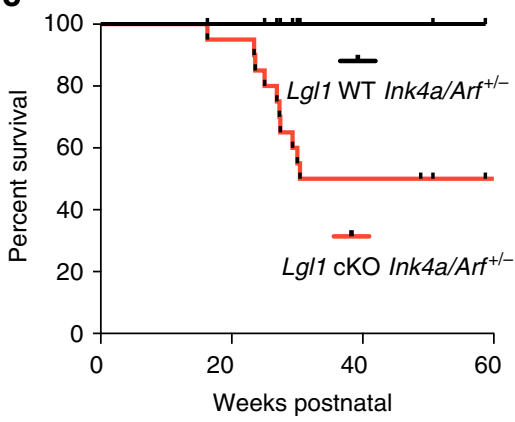

e

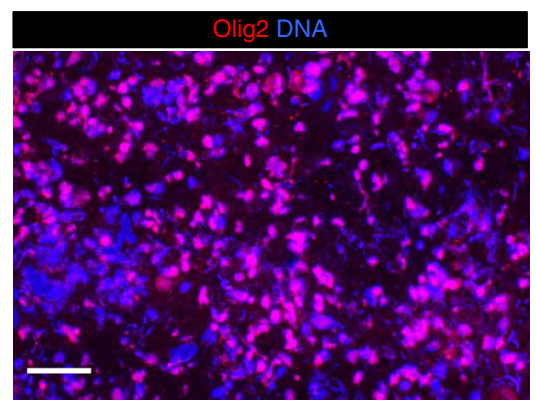

d

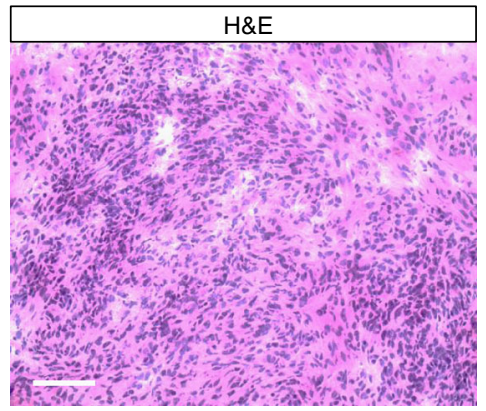

f

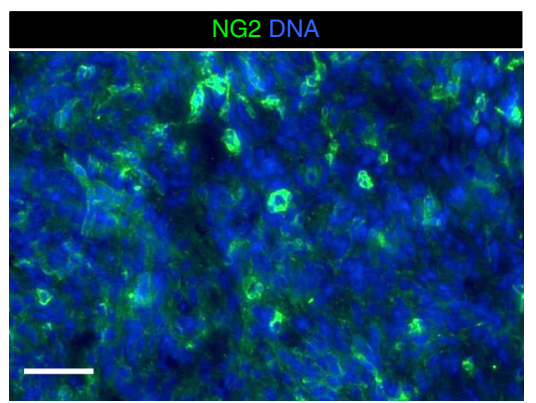

Fig. 4 Lg/1 knockout increases proliferation and promotes gliomagenesis. a Representative immunostaining of corpus callosum of $\mathrm{Lg} / 1 \mathrm{WT}$ mouse (top panel) and Lgl1 cKO mouse (bottom panel) for Tom (red) and co-staining for DNA (gray) after broad deletion of Lgl1 in NG2-CreERT2 Tom Lgl1 WT (top panel) vs. Lg/1 cKO P30 mice followed by analyses at P60 by FACS and IF. Scale bar: $200 \mu \mathrm{m}$. b Quantification of Tom+ cells by FACS from Lg/1 WT and $\mathrm{Lg} / 1 \mathrm{cKO}$ corpus callosum, showing a significant increase following Lgl1 knockout. Data are represented as mean \pm s.e.m. from the analyses of $5 \mathrm{Lg} / 1 \mathrm{WT}$ vs. 5 Lgl1 cKO mice ( ${ }^{\star \star *} p<0.001$, Mann-Whitney test). c Kaplan- Meier survival curve for Lgl1 WT (Lg/7wt/wt; NG2Cre; Ink4a/Arf ${ }^{+/-}$) and NG2Cre-driven homozygous $\mathrm{Lg} / 1 \mathrm{cKO}\left(\mathrm{Lg} / \mathrm{fl}_{\mathrm{flfl}}\right.$; NG2Cre; Ink4a/Arf $\mathrm{N}^{-}$) mice. Six animals were analyzed for each genotype. d Hematoxylin and Eosin (H\&E) staining of endogenous tumors in $\mathrm{Lg} / 1 \mathrm{CKO}$ mice, showing infiltrative growth. Scale bar: $100 \mu \mathrm{m}$. e, $\mathbf{f}$ Immunostaining of endogenous tumors in $\mathrm{Lg} / 1 \mathrm{NG2-Cre} \mathrm{mice} \mathrm{for}$ Olig2 (blue, e) and NG2 (green, f). Scale bar: $50 \mu \mathrm{m}$ 
(Supplementary Figs. $4 \mathrm{~d}-\mathrm{f}$ ). These data indicate that Lgl1 restricts proliferation of OPC in the healthy and demyelinated CC.

Increased proliferation, defective differentiation, and decreased rates of ACD are associated with gliomagenesis ${ }^{2}$. We therefore tested whether Lgl1 knockout promotes tumor formation. Immunostainings of normal adult mouse brain revealed that OL express the tumor suppressor p19Arf (Supplementary Fig. 5a) ${ }^{36}$. We therefore bred Lgl1 floxed mice ${ }^{21}$ with a NG2-Cre driver line ${ }^{37}$, and mice heterozygous for the Ink4a/Arf deletion (Ink4a/Arf $+/-$ ) 38 . Mice were monitored for neurological signs of tumor formation and survival. We observed that $100 \%$ of $L g l 1$ WT Ink4a/Arf $+/-$ mice were still alive at 60 weeks postnatal age, whereas Lgll cKO Ink4a/Arf $+/$ - mice had a significantly shorter median survival of 45.79 weeks (Fig. 4c; $p=0.0108$ ). Histopathologic analyses of Lgl1 cKO Ink4a/Arft/- brains showed infiltrative tumors consistent with astrocytoma morphology (Fig. 4d). Expression analysis by qrtPCR in tumor cells isolates showed a strong reduction of $p 19^{A r f}$ mRNA versus Lgll cKO Ink4a/Arf WT and Ink4a/Arf+/- cells, suggesting that the WT allele of $p 19 A r f$ is not expressed in the tumor (Supplementary Fig. 5b). Fluorescent immunostainings of brain sections from tumor-bearing mice showed that tumors express the oligodendrocyte lineage and glioma markers Olig2 and NG2. Results are indicative of a mixed astrocytic and oligodendroglial high-grade glioma (Figs. 4d-f) ${ }^{39}$.

To determine whether our animal model findings were clinically relevant, we mined expression data from 99 patients from The Cancer Genome Atlas (TCGA) for a potential association of Lgl1 mRNA expression levels and glioblastoma patient survival. We found that low levels of Lgl1 mRNA expression correlated with poor patient survival (Supplementary Fig. $5 c)^{40}$. In further support of a tumor suppressive function, Lgl1 protein expression was absent in six out of seven human glioblastoma xenografts as shown by immunoblotting (Supplementary Fig. 5d). Collectively, these data showed that Lgl1 attenuates OPC proliferation and synergizes with tumor suppressor Ink4a/Arf to suppress gliomagenesis.

Lgl1 cKO OPC show a defect in NG2 routing to the lysosome. A recent study showed that levels of surface NG2 are regulated by endocytosis $^{15}$. We hypothesize that Lgl1 cKO OPC fail to route NG2 to the lysosome and thereby sustain aberrantly high NG2 levels. In order to test this, we transfected Lgll cKO and WT cells with lysotracker GFP (Lys-GFP) and labeled cell surface NG2 with an antibody specific for its extracellular domain (NG2-EC) and a fluorescent secondary antibody. Cells were consecutively monitored by fluorescent time-lapse microscopy for up to three hours under differentiation conditions. In both Lgl1 cKO and WT OPC, NG2-EC started to reach the lysosome one hour post imaging (Fig. 5a). Co-localization of NG2-EC with Lys-GFP peaked in Lgll WT cells three hours after starting the imaging. Interestingly, we observed a significantly higher colocalization of NG2 with lysosomes in WT OPC compared to Lgll cKO OPC (WT $=79.6 \%$; $c K O=54.7 \%, p=0.0047$ ) (Fig. 5a)

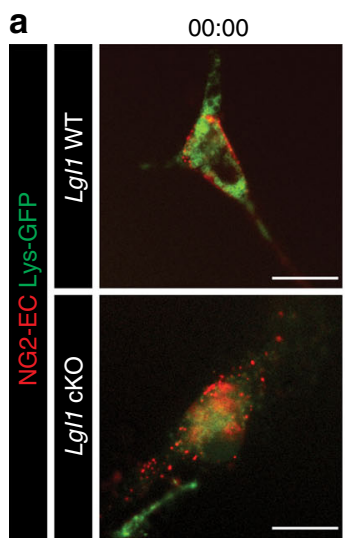

b

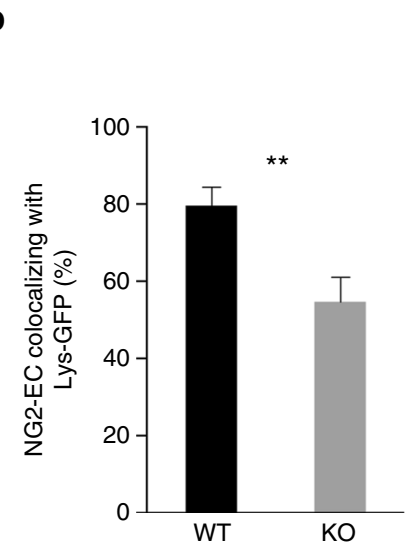

01:00

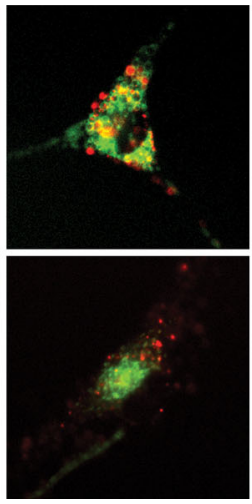

C

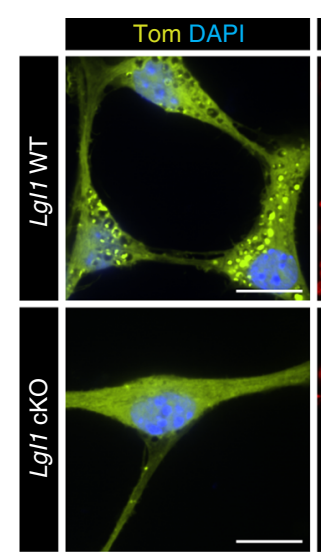

02:00
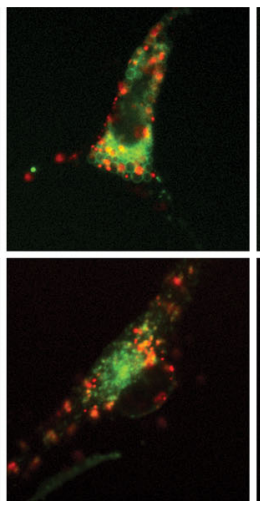
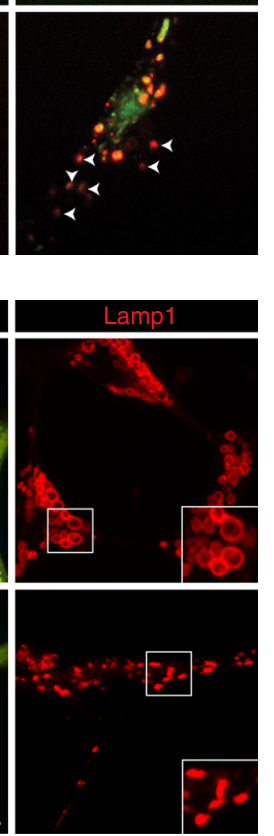

Fig. 5 Lg/1 CKO OPC show defect in NG2 routing to the lysosome. a Time-lapse fluorescent pictures of Lg/7 WT and Lg/7 cKO OPC labeled for NG2-EC (red) and lysosome (Lys-GFP, green) under differentiation conditions. Pictures are acquired every hour for 3 hours. White arrows indicate NG2-EC that did not reach the lysosome. Time indicated as hours:minutes. Scale bar: $25 \mu \mathrm{m}$. b Quantitative graph representing the ratio of labeled NG2 puncta in the lysosomes in WT (black) and Lg/1 cKO cells (gray). Data are represented as mean \pm s.e.m., $n=10$ cells each genotype, three independent experiments (** $p<0.01$, Student's t-test). c Immunostaining of NG2dTom cells (yellow) with lysosomal protein Lamp1 (red) showing the difference in lysosome shape between Lg/1 WT OPC and cKO OPC. Scale bar: $25 \mu \mathrm{m}$ 
suggesting that in differentiating OPC, Lgl1 acts as a positive regulator of NG2 trafficking to the lysosome. These results prompted us to stain for the lysosome associated membrane protein 1 (Lamp1) to investigate whether the lysosome is formed properly in Lgl1 knockout cells. We find that lysosomes are present in Lgl1 cKO OPC, based on Lamp1 positivity and subcellular localization. Noteworthy, lysosome shape appears to be altered due to Lgl1 knockout (Fig. 5c). Collectively, these results show that Lgl1 positively regulates NG2 trafficking to the lysosome.
Non-degraded NG2 in Lgl1 cKO OPC is recycled to the membrane. Given that NG2 is a transmembrane proteoglycan ${ }^{41}$, we suggest that it might be recycled to the membrane by the recycling endosome, a tubular structure continuous with the early endosome. To test this, we incubated Lgl1 cKO cells in differentiating conditions and live labeled NG2-EC. Cells were fixed at different time points after surface NG2 labeling and stained for recycling protein markers. At $45 \mathrm{~min}$, we observed a higher colocalization of NG2-EC with the recycling regulator Rab11 in Lgll cKO compared to WT cells (Fig. 6a). At $24 \mathrm{~h}$, NG2-EC a
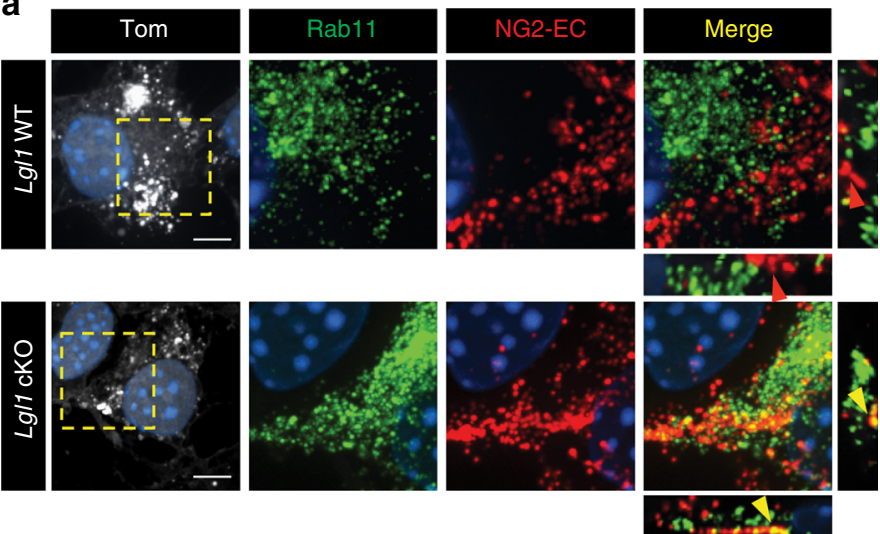

d
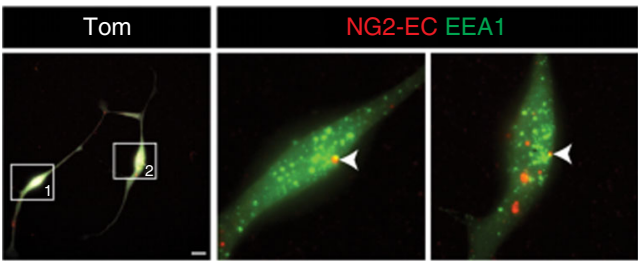

$\mathbf{f}$
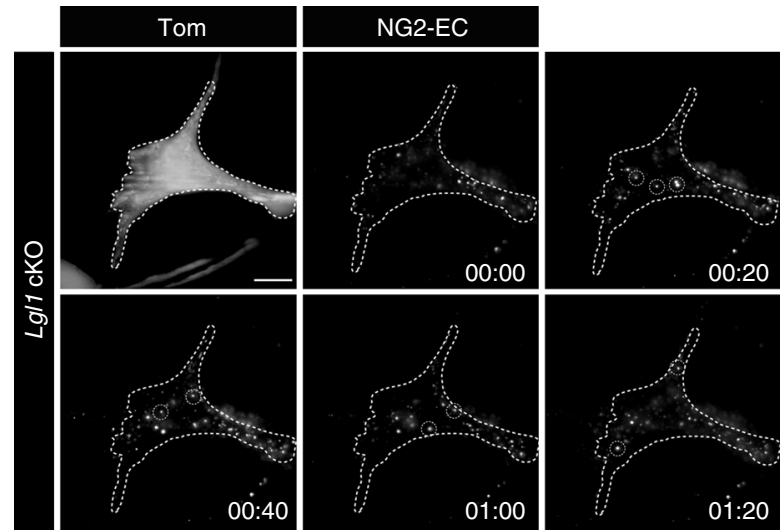

b
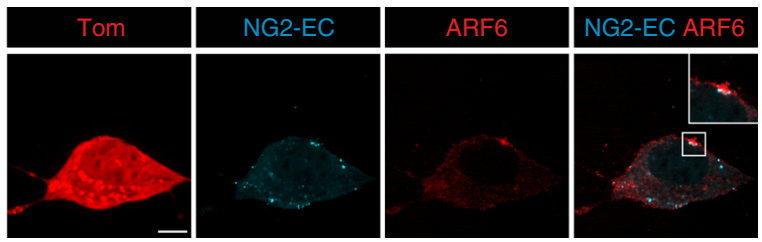

C
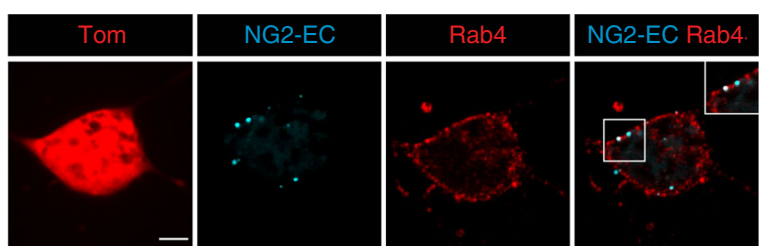

e

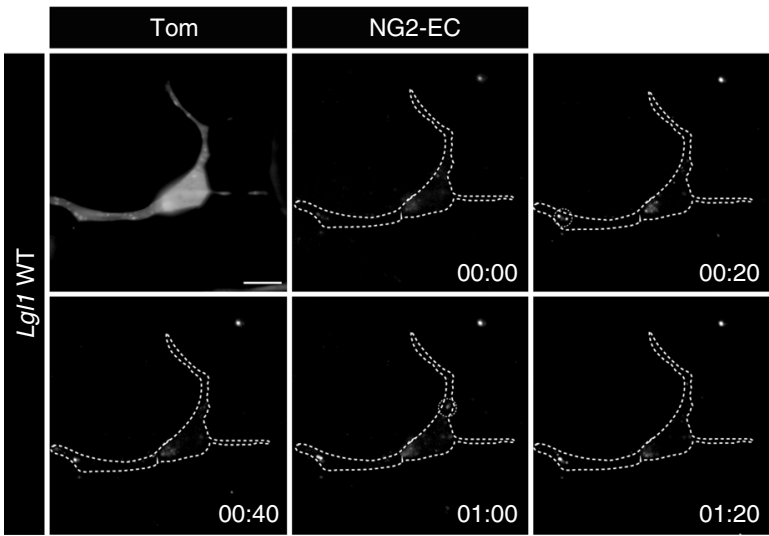

g

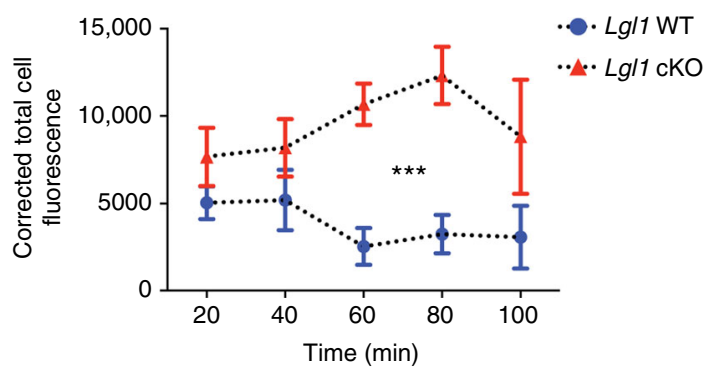

Fig. 6 Non-degraded NG2 in Lg/1 cKO OPC is recycled to the membrane. a Representative co-immunostaining for NG2-EC (red) and Rab11 (green) 45 min after incubation in differentiation medium. Note the higher colocalization of NG2-EC with Rab11 in Lgl1 cKO OPC (yellow arrowheads) compared to Lg/1 WT cells (red arrowhead). Scale bar: $5 \mu \mathrm{m}$. b-d Fluorescent immunostaining pictures illustrating colocalization of remaining NG2 (blue) with (b) ARF6 (red), (c) Rab4 (red), and (d) EEA1 (green) in Lgl1 cKO cells 24 hours after incubation in differentiation conditions. Scale bars: $10 \mu \mathrm{m}$. e, $\mathbf{f}$ Frames illustrating TIRF time lapse of $L g / 1 \mathrm{cKO}$ and WT cells 4 hours after incubation in differentiation conditions. Pictures were taken each 20 min. Examples of recycling labeled NG2 puncta (white) are highlighted in dashed circle. NG2 recycling persists in Lg/1 cKO cells up to time point 01:20 whereas it is insignificant in WT cells. Time indicated as hours:minutes. Scale bar: $10 \mu \mathrm{m}$. $\mathbf{g}$ Graph representing quantification of the corrected total cell fluorescence reflecting NG2 recycling in Lg/1 cKO cells (blue circles) and in Lg/1 WT cells (red lozenges). Data are depicted as mean \pm s.e.m., $n=8$ cells for each genotype, three independent experiments $\left({ }^{\star \star *} p<0.01\right.$, two-way ANOVA) 
colocalizes with ARF6, a GTPase critical for endocytic recycling, supporting the notion that NG2 is recycled to the membrane in Lgl1 cKO OPC (Fig. 6b). Additionally, NG2-EC overlaps with Rab4, a GTPase marker for fast endocytic recycling, hinting that NG2 recycling may not be restricted to the slow recycling pathway (Fig. 6c). We also observed co-localization of NG2-EC with the early endosome marker EEA1 (Fig. 6d), suggesting that NG2 is constantly uptaken and shuffled back to the membrane. Noteworthy, we observed a coexpression of Lgll and the early endosome-specific protein Rab10 both at the membrane and around the nucleus, further associating Lgl1 role with the endocytic machinery ${ }^{42}$ (Supplementary Fig. 6).

In order to better investigate a potential NG2 recycling in Lgll cKO cells, we used total internal reflection fluorescence (TIRF) to monitor live-labeled NG2-EC docking to the membrane. Cells were plated in differentiation medium for four hours and then monitored. By this time, the majority of NG2 is degraded in Lgll WT cells and no significant NG2 recycling was observed (Fig. 6e,). To the contrary, NG2-EC was more abundant and continuously re-introduced to the membrane in Lgl1 cKO OPC (Figs. 6f, g; Supplementary Movie 1 see Methods).

Collectively, these data proof that in Lgll cKO cells, NG2 is mostly recycled to the membrane rather than degraded.

Lgl1 cKO OPC show premature and accelerated endocytosis recycling of NG2. To better characterize the deregulation of endocytosis in Lgll knockout cells, we acutely isolated Tom+ cells from Lgl1 NG2-Cre ${ }^{E R T 2}$ Ai14-TdTom Lgl1 flffl and Lgl1 ${ }^{\text {wt } / w t}$ control mice injected with tamoxifen at P30. Fifteen days after starting Lgll knockout, gene expression was analyzed using highsensitivity, whole-transcript arrays (Fig. 7a; see also Methods). By analyzing the 318 genes differentially expressed between $L g l 1$ WT and Lgll cKO OPC $(p<0.05)$, ingenuity pathway analysis identified several pathways including the receptor-mediated endocytosis pathway to be upregulated in Lgll cKO cells (Fig. 7b; activation $z$-score $=2, p=0.018$ ). Upregulation of early endocytosis genes Ap2a1, Ep4112, Hip1R, EEa1, Reps1, Syne1, and Pdl1 was further validated by qrt-PCR in Lgll cKO OPC vs. Lgll WT OPC (Fig. 7c). These data suggest that Lgll knockout deregulates endocytic uptake in addition to endocytic recycling.

In order to investigate if early endocytosis is affected in Lgl1 cKO cells, we labeled surface NG2 with the NG2-EC antibody, and marked the early endosome by expression of Rab5a-GFP. Time-lapse imaging of cells for $30 \mathrm{~min}$ in differentiation conditions showed a prominent colocalization of NG2 with Rab5a only in mutant cells, suggesting that Lgll negatively regulates NG2 uptake (Fig. 7d). We corroborated these results by quantifying the endocytosis of transferrin using FACS, and found a ten-fold increase in transferrin incorporation in Lgll cKO OPC as compared to Lgl1 WT OPC (Supplementary Fig. 7).

Next, we tested whether NG2 is only recycled after failing to be degraded or also when prematurely internalized in Lgll cKO OPC. We used TIRF to monitor labeled NG2 docking to the membrane over the first $5 \mathrm{~min}$ of differentiation conditions. We observed that a significantly higher ratio of labeled NG2 was endocytosed and recycled to the membrane in Lgll cKO cells compared to WT cells (WT $=15 \%$; $\mathrm{cKO}=58 \%, p<0.0001$ ) (Figs. $7 \mathrm{e}-\mathrm{g}$ ). Taken together, these data show that Lgl1 negatively regulates NG2 internalization and recycling and establish Lgl1 as a regulator of NG2 trafficking by the endocytic pathway.

Differentiation defect in $\mathbf{L g l l} \mathrm{cKO}$ OPC is linked to aberrant NG2 recycling. Our studies above suggest that Lgl1 knockout enhances NG2 internalization and recycling to the membrane, thereby preventing NG2 degradation and blocking OL differentiation. In order to dissect whether abnormal NG2 recycling has an effect on OL differentiation, Lgll cKO cells were incubated for 4 hours with monensin $(10 \mu \mathrm{M})$, a drug widely used to interrupt protein recycling ${ }^{43}$. We tested if the drug has a specific effect on NG2 recycling in $\mathrm{Lgll}$ cKO cells by monitoring Tom+ cells under TIRF for up to one hour after monensin treatment. We observed that monensin treatment blocked NG2 recycling, validating the reported drug effect on recycling (Fig. 8a) ${ }^{43}$. Lastly, we assessed differentiation in treated and non-treated $\mathrm{Lgll} \mathrm{cKO}$ cells kept in differentiation conditions for 5 days by O4 and NG2 co-immunostaining. A quantification of marker-positive cells showed that monensin treatment significantly increased the ratio of $\mathrm{O} 4+$ cells at the expense of NG2 + cells (treated/O4 $+=75 \%$ and NG2 $+=24 \%$; non-treated $/ \mathrm{O} 4+=9 \%$ and NG2 $+=90 \%$; $p<0.0001$ ) (Figs. 8b, c).

These results link abnormal recycling of surface NG2 and defective OL differentiation.

\section{Discussion}

Adult OPC differentiate in multiple steps, beginning by committing to OL fate and ending by maturing and producing myelin. There is a wealth of studies on the later steps of OL differentiation and maturation ${ }^{34,44}$. Little is known, however, about the earliest decisions between OPC and OL fate. Thus far, the downregulation of proteoglycan NG2 is the earliest immunophenotypic change that distinguishes the two daughter cells within a newly formed OPC pair upon ACD. NG2 marks the OPC daughter, while the NG2-negative daughter acquires $\mathrm{CC} 1$ expression, which marks OL differentiation 23,9 . NG2 knockout OPC show delayed proliferation, which indicates that NG2 controls the timing of OPC proliferation ${ }^{7}$ (reviewed by Sakry et $\mathrm{al}^{45}$ ). The NG2 knockout does not fully block OPC proliferation and differentiation and its effect is only temporary ${ }^{7}$. Thus, downregulation of NG2 is an important but not the only step for OL commitment. Indeed, PDGFRa is also asymmetrically distributed amongst newly formed OPC pairs ${ }^{3}$, further suggesting that cell fate determinants in addition to NG2 link ACD to differentiation ${ }^{46}$.

Due to the reported functional, morphologic, and neuroanatomical heterogeneity of $\mathrm{OPC}^{47}$, and its potential influence on cell division mode, cell cycle lengths, and differentiation ${ }^{1,9,48,49}$, we focused our studies on OPC in the corpus callosum. We analyzed OPC in mice between the ages P30 and P60 with the exception of tumor-bearing mice, which were older. The rationale for analyzing OPC at these stages was that the analyses of mice older than P60 would be technically very difficult due to a long OPC cell cycle time ${ }^{1}$. We expect to detect the maximum number of ACD between P30 and P60, because the rates of asymmetric OPC divisions are already high at postnatal stages $(\mathrm{P} 20 ; \mathrm{ACD}=\sim 35 \%)$ and peak at $\mathrm{P} 60(\mathrm{ACD}=\sim 45 \%)$ before decreasing again with age $(\mathrm{P} 120 ; \mathrm{ACD}=\sim 35 \%)^{3}$. Moreover, the rate of differentiation remains stable at $40 \%$ between $\mathrm{P} 30$ and $\mathrm{P} 60^{9}$, which makes is a suitable range to identify potential effects on differentiation of a single gene knockout.

Since ACD generate differentiated cells from stem and progenitor cells across species, it was attractive to speculate that conserved regulators of ACD, such as Lgl1, regulate early steps in OL differentiation. Previous studies showed that Lgll localizes to the lateral membranes and cytoplasm of embryonic neural progenitor cells $\mathrm{s}^{21,22}$. Expression of Lgl1 protein in rat neocortex homogenates persists into adulthood ${ }^{42}$. Functional analyses in the adult brain, however, were limited to its role in SVZ neural stem cells, neurons, and astrocytes ${ }^{21-23,42}$.

Here, we show that $L g l 1$ cKO cells fail to lose NG2 expression and exhibit differentiation defects. Interestingly, we find that $L g l 1$ 
a

b

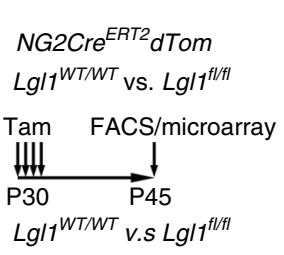

d
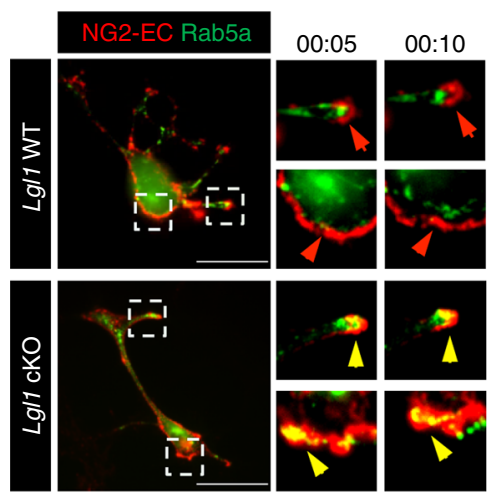

f

\section{NG2-EC}
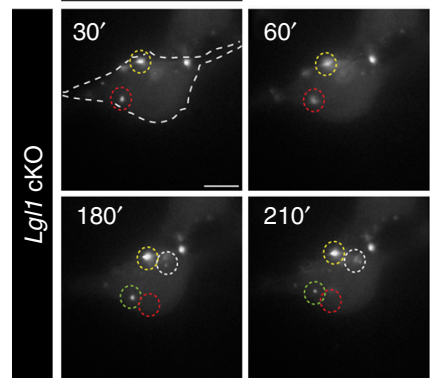
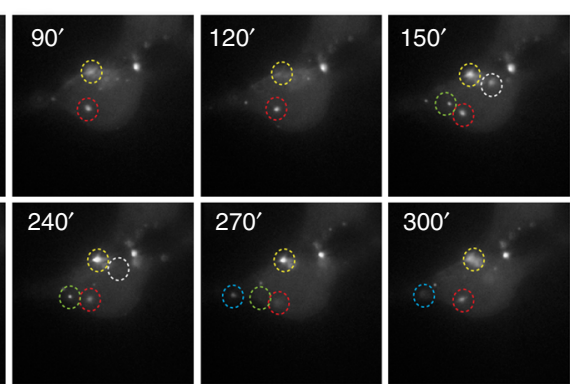

g

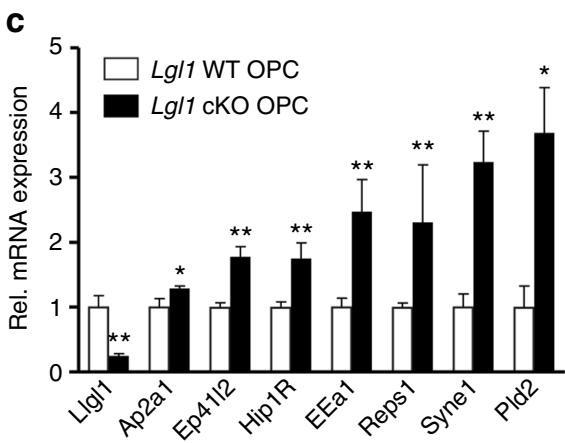

e
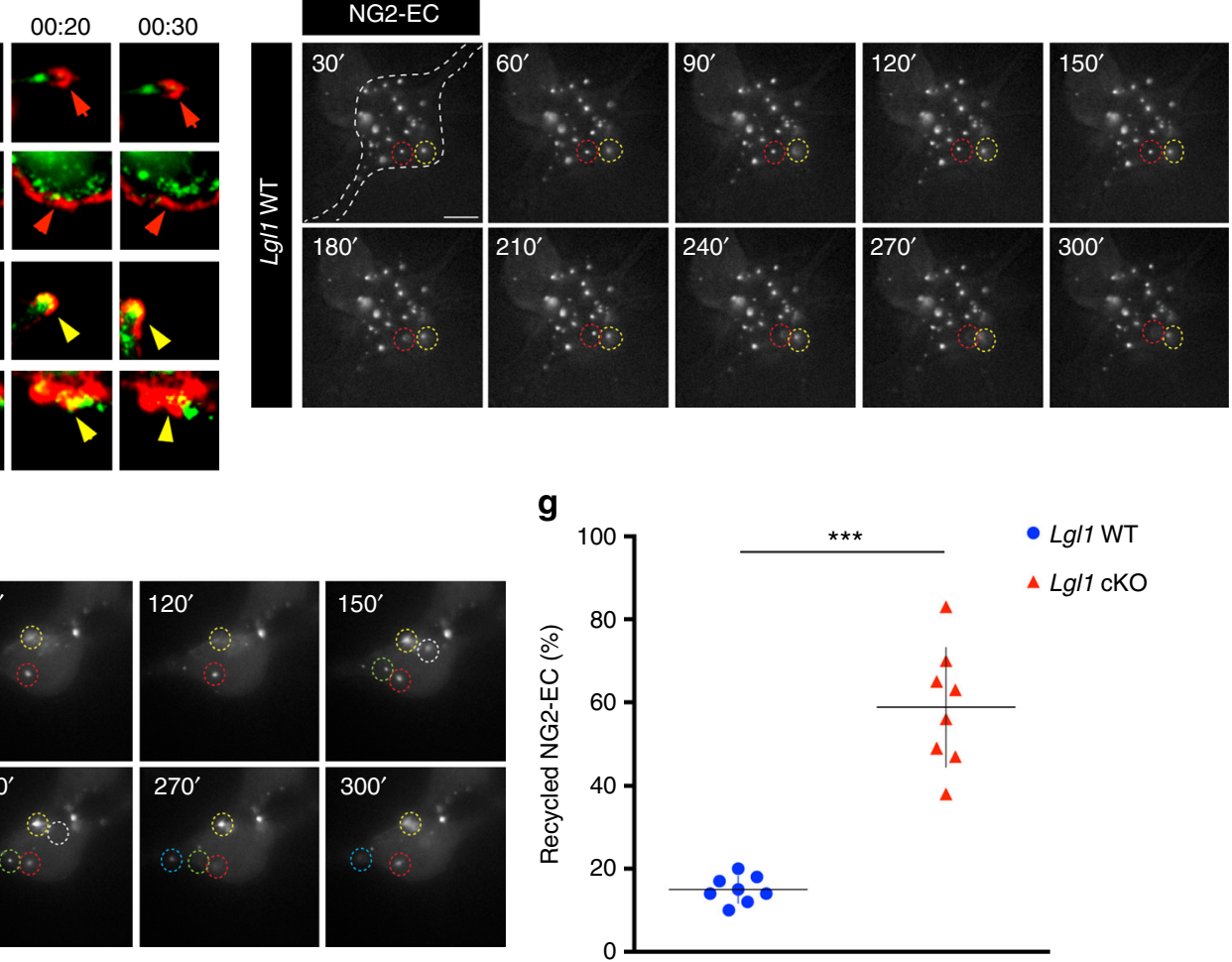

Fig. $7 \mathrm{Lg} \mid 1$ negatively regulates internalization and recycling of NG2 in OPC. a Schematic illustration of the experimental approach assessing global changes in gene expression by microarray in Lg/7 WT vs. Lg/1 cKO OPC. FACS sorted red fluorescent PDGFR $\alpha+04-$ cells. b Bar graph of ingenuity pathway analyses (IPA) of Lg/7 WT vs. CKO OPC. Receptor-mediated endocytosis pathway is amongst the upregulated pathways in $L g / 1 \mathrm{cKO}$ cells. $\mathbf{c}$ Validation by q-RT-PCR for mRNA encoding receptor-mediated endocytosis regulators that were differentially expressed in $L g / 1 \mathrm{cKO}$ cells as determined by microarray analyses. OPC were derived from the corpus callosum of GFAP-Cre, Tom, $L g / 7^{w t / w t}$ and, GFAP-Cre, Tom, Lg/fl/fl mice. Data represented as mean \pm s.e.m. $n=6 \mathrm{Lg} / 1 \mathrm{WT}$ and $6 \mathrm{Lg} / 1 \mathrm{cKO}$ animals $\left({ }^{\star} p<0.05,{ }^{* \star} p<0.01\right.$, Mann-Whitney test). $\mathbf{d}$ Steady-state frames of time lapse (1 frame/ $\left.10 \mathrm{~min}\right)$ of NG2-EC (red) and early-endosome marker Rab5a (green) in Lg/7 cKO and WT OPC. Note that NG2 stays mostly at the membrane in Lg/7 WT cells (red arrows), whereas it co-localizes with the early endosome in the cytoplasm of $L g l 1 \mathrm{cKO}$ OPC (yellow arrows). Scale bar: $20 \mu \mathrm{m}$. e, $\mathbf{f}$ Frames illustrating time lapse experiment under TIRF microscopy. Pictures are acquired each 30 seconds as soon as cells were exposed to differentiation conditions. Each white puncta in focus represents labeled NG2-EC docked at the membrane. Individual recycled puncta are highlighted with dashed circles of different colors. Note that in $\mathrm{Lg} / 1$ cKO cells, the majority of the labeled NG2 is internalized and recycled to the membrane whereas in WT cells, the majority of NG2 puncta retains a stable localization at the membrane. Scale bars: $10 \mu \mathrm{m}$. $\mathbf{g}$ Graph representing the quantification of NG2 recycling at the membrane throughout the first 5 min of differentiation conditions. Data represented as mean \pm s.e.m. $n=3$ cells per genotype, three independent experiments $\left({ }^{\star \star \star} p<0.005\right.$, Student's $t$-test)

knockout in OPC increases the rates of cells in a transitional state and expressing both OPC marker NG2 and OL marker CC1, at the expense of mature OL. These data indicated that Lgl1 loss does not prevent the expression of $\mathrm{CC} 1$ but rather prevents downregulation of NG2, and thereby permanently attenuates differentiation.

Whereas normal OPC populate the brain sparsely and show little overlap in the areas that two adjacent OPCs occupy ${ }^{50}$, they are denser in demyelinated lesions, such as those typically found in chronic inflammation models in mice and in multiple sclerosis patients $^{32,33}$. We found that Lgl1 is a critical regulator of OL differentiation during remyelination following chemical-induced demyelination. OPC are considered a critical source for remyelination and failed OPC differentiation are thought to be one important underlying cause for failed remyelination in chronically demyelinated lesions ${ }^{51}$. It is interesting to speculate that Lgl1 functions at an early step in the complex regulatory process of remyelination and thus may be a viable target for approaches aimed at promoting proper differentiation of OPCs in demyelinated lesions. 
a
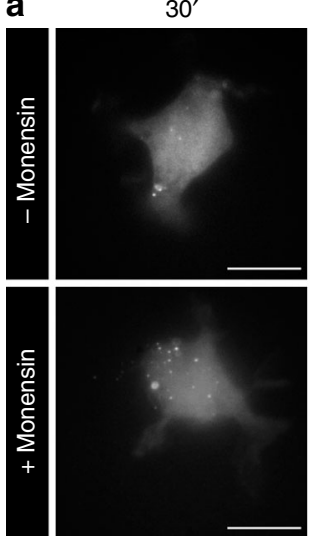

b

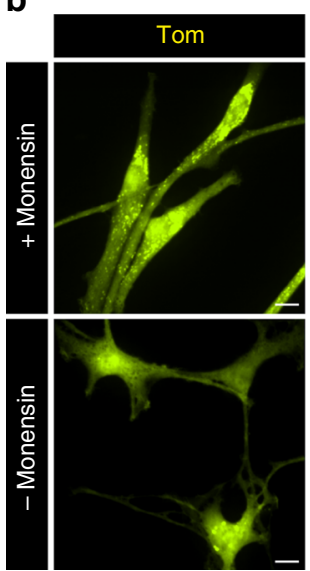

$60^{\prime}$
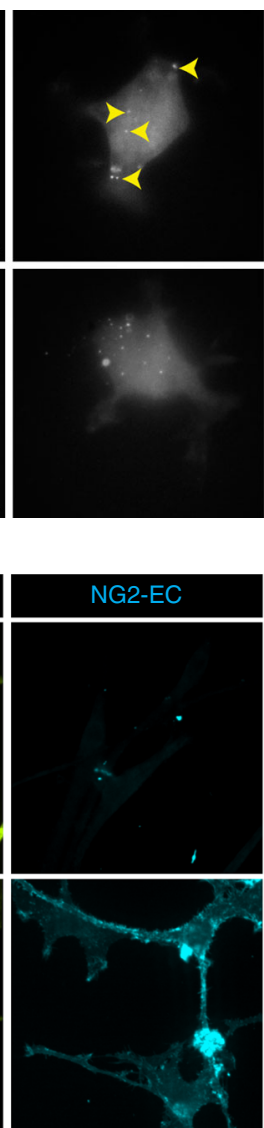

$90^{\prime}$
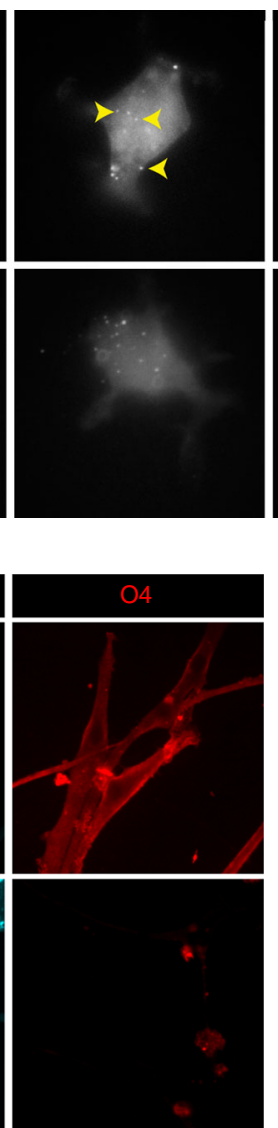

$120^{\prime}$

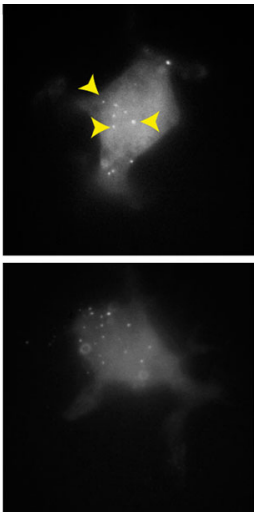

C

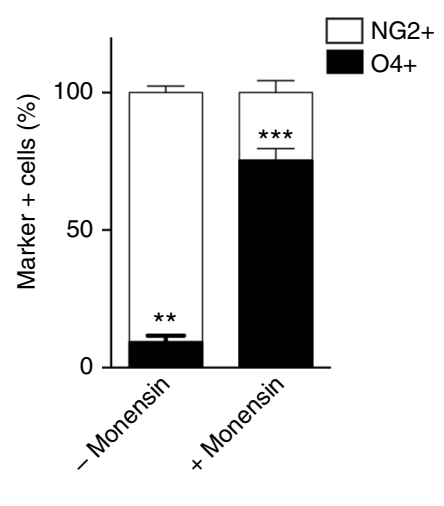

Fig. 8 Aberrant NG2 recycling is linked to differentiation defects. a Steady-state images of two single Lgl1 cKO cells monitored for NG2-EC (white dots) by TIRF, treated (bottom panel) or not treated (upper panel) with monensin. Pictures are acquired each 30 seconds. Note that NG2 is recycled to the membrane in non-treated cells (yellow arrow), whereas no recycling is observed in treated cells. Scale bar: $10 \mu \mathrm{m}$. b Fluorescent pictures illustrating Lg/7 cKO cells (Tom) monensin -treated or untreated and labeled for NG2 (blue) and O4 (red) after 5 days in differentiation conditions. Scale bar: $10 \mu \mathrm{m}$. c Graph representing quantification of $\mathrm{O} 4+$ and NG2+ cells in monensin-treated or untreated Lg/1 cKO cells. Untreated cells are mostly NG2+ and O4-, whereas treated cells show a reverse phenotype with a higher number of cells expressing O4. Data are depicted as mean \pm s.e.m., $n=20$ cells per genotype, three independent experiments $\left({ }^{\star \star} p<0.01\right.$, Student's $t$-test)

Our analyses of cell division modes support the hypothesis that Lgl1 restricts NG2 during ACD as part of the mechanism to turn on OL differentiation in the NG2-negative progeny. Moreover, Lgl1 does not disrupt symmetric, differentiating divisions, which suggests that it specifically regulates differentiation in the ACD progeny. To date, only a few conserved ACD regulators have a known function in OL differentiation in addition to Lgl1. These include the polarity regulator partitioning-defective 3 (Par3) protein, which promotes myelination in the peripheral nervous system $^{52}$, and the transcriptional regulator Prox1, which is required for OL lineage in the mouse. Par3, Prox1, and Lgl1 are in the same protein network that controls ACD in Drosophila neuroblasts ${ }^{46}$. A role for Par3 and Prox1 in ACD of OPC has yet to be determined.

Multiple studies have shown that $\mathrm{OPC}^{13,14}$ and $\mathrm{OL}^{36}$ are a cellular origin of glioma in genetically engineered mouse models. The neoplastic transformation of OPC and OL and formation of gliomas are associated with increased proliferation, decreased differentiation, changes in the tumor microenvironment ${ }^{53}$, and a decrease in $\mathrm{ACD}^{2}$. Lgll knockout during embryonic neurogenesis disrupts the asymmetric division of neuroepithelial cells and generates rosette-like structures reminiscent of PNETs but perinatal death prevented the analyses of tumor formation ${ }^{21}$. Lgl1 knockout in cell types known to only undergo symmetric but not asymmetric divisions, such as astrocytes, did not generate premalignant lesions ${ }^{23}$. Taken together with our data, these findings suggest that defective ACD might be an underlying cause for proliferation and differentiation defects in glioma cells. Our data support the notion previously observed in epithelial cells ${ }^{54}$ that loss of polarity-albeit by itself not sufficient to induce carcinogenesis-contributes to tumor formation. We show that Lgl1 loss synergizes with Ink4a/Arf loss, which is a frequent and early event in glioma ${ }^{24}$, to induce gliomagenesis (Fig. 4). There is strong precedence for Ink4a/Arf heterozygous mice to be as susceptible as Ink4a/Arf nullizygous mice to gliomagenesis ${ }^{55}$. A likely explanation for this is, that the remaining Ink4a/Arf allele in tumors arising from Ink $4 a / A r f+/-$ mice is inactivated at high frequency, which is supported by our findings that p19Arf mRNA expression is greatly reduced in Lgll KO tumors. Although 19Arf and $\mathrm{p} 16^{\text {Ink }}$ transcripts are reportedly low in OPC and OL, we find overlapping expression of the p19Arf protein and OL marker CC1 (Supplementary Fig. 5). This is interesting, because p19Arf but not $16^{\text {Ink }}$ is an important tumor suppressor in a glioma mouse model study ${ }^{36}$. Collectively, the data suggest that tumor formation in NG2-Cre Lgl1 KO Ink4a/Arf +/- mice required inactivation of p19Arf expression, hence the long latency (Supplementary Fig. 5). It is also plausible that Lgl1 suppresses tumor formation by restricting NG2 function as a co-receptor for receptor tyrosine kinases, since deregulated PDGFRa and EGFR signaling activity contributes to gliomagenesis ${ }^{40,56}$ (for review see ref. ${ }^{57}$ ). 
Endocytosis has a range of functions including regulating the cell surface expression and subcellular localization of proteins, remodeling the membrane and controlling signaling pathways ${ }^{16}$. Studies in Drosophila epithelia cells support a link of polarized protein localization and endocytosis by showing that loss-offunction mutants of the critical endosome adaptor AP2 leads to defects in polarity and differentiation similar to those observed in Lgl loss-of-function experiments ${ }^{58}$. Our study proposes an unprecedented link between defects in NG2 endocytic trafficking, $\mathrm{ACD}$, and differentiation. Indeed, in Lgl1 cKO mutant cells, we find that NG2 is not properly routed to the lysosome and instead is recycled to the membrane. These observations show that endocytic pathway defects in Lgll cKO OPC disturb the regular intracellular itinerary of NG2 during differentiation and lead to its recycling rather than degradation. NG2 levels are permanently suppressed in OL most likely as a result of downregulation of the NG2 promoter in the OL daughter after $\mathrm{ACD}^{3}$.

Taken together, these data suggest that the unequal distribution of NG2 in the newly formed progeny of ACD is achieved by NG2 degradation in the lysosome and is maintained by silencing the NG2 promoter in the OL daughter.

We can only speculate as to whether Lgl1 regulates NG2 trafficking directly or, indirectly, by regulating the endocytic machinery. Although not previously implicated as a negative regulator of endocytosis in mammalian cells, Lgll activates endocytosis protein and GTPase Rab10 by releasing the GDP dissociation inhibitor (GDI) in rat hippocampus to induce polarized axon growth ${ }^{42}$. In cells undergoing $\mathrm{ACD}$, such as Drosophila neuroblasts, Lgl1 establishes cell polarity by mechanisms that regulate myosin II activity, directed vesicle transport and the subcellular localization of proteins (for review see ref. ${ }^{46}$ ). Nonmuscle myosin II (NMII) is a critical regulator of clathrin-mediated endocytosis ${ }^{59}$ and is a negative regulator of oligodendrocyte morphological differentiation ${ }^{60}$. Lgl1 associates with NMII and inhibits filament formation ${ }^{61}$ and reduces the association of NMII with actin. Whether Lgll regulates endocytosis by affecting motor protein dynamics or through other mechanisms, such as regulating the subcellular localization of the GTPases $^{62,63}$, remains to be determined. In further support of an indirect effect of Lgl1 on endocytic trafficking, Drosophila Lgl1 regulates vesicle acidification in the lysosome ${ }^{64,65}$. Noteworthy, our data indicate that lysosomes in $\mathrm{Lgll}$ cKO OPC have a tubular shape rather than the vesicular shape seen in WT OPC, hinting that lysosome maturation might be affected by Lgll knockout (Fig. 5).

Future studies should investigate further the potential link of defects in endocytosis and gliomagenesis.

\begin{abstract}
Methods
Mice. All mouse experiments were approved by and performed according to the guidelines of the Institutional Animal Care and Use Committee of the University of California, San Francisco. Lgl1 lox/lox mice were courtesy of Dr. Vasioukhin ${ }^{21}$ and were bred with NG2-Cre (Jax strain 008533) ${ }^{37}$ and GFAP-Cre (Jax strain 004600) ${ }^{66}$, NG2-CreER ${ }^{T M}$ (Jax strain 008583) ${ }^{9}$ and Ail4 Cre reporter mice (Jax strain 007908) ${ }^{29}$ mice or Ink4a/Arf hemizygous knockout mice ${ }^{38}$. AscllGFP ${ }^{67}$ and NG2DsRed ${ }^{37}$ mice from Jackson Laboratories were bred together to obtain Ascl1GFP-NG2DsRed double transgenic mice. FVB/N mice were obtained from Simonsen Laboratories, Gilroy. For survival studies, Lgll cre-conditional deleted mice were kept under observation until reaching the major endpoint, which was animal neurological symptom-free survival.
\end{abstract}

Induction of $\boldsymbol{L} g / \mathbf{1}$ knockout and demyelination. Tamoxifen (Sigma-Aldrich) was dissolved in corn oil (Sigma-Aldrich) at $20 \mathrm{mg} / \mathrm{mL}$ and injected intraperitoneally (i.p.) at $1 \mathrm{mg}$ to induce Cre activity La-lysophosphatidylcholine (lysolecithin = LPC, Sigma-Aldrich) was dissolved at $1 \%$ in physiological serum and $0.5 \mu \mathrm{l}$ were injected to induce demyelination. For clonal labeling of NG2 cells P30-P52 NG2$C r e^{E R T 2}$, TdTom, Lgl flflfl and NG2-Cre ${ }^{E R T 2}$, TdTom, $L g l l^{\text {wt/wt }}$ mice were injected with $1 \mathrm{mg}$ tamoxifen twice within $24 \mathrm{~h}^{35}$. To assess proliferation rates and cell division modes of OPC, mice were subsequently treated with $0.1 \mathrm{mg} / \mathrm{mL}$ EdU in drinking water for 14 days and resected brains were processed for immunofluorescence. For differentiation NG2-Cre ${ }^{E R T 2}$, TdTom, Lgl1 $1^{f l / l}$ and NG2-Cre ${ }^{E R T 2}$, TdTom, Lgl1 ${ }^{w t / w t}$ mice were injected with tamoxifen either once or twice daily for 4 consecutive days. Mice were analyzed 30 days post injection.

For broader deletion of Lgl1, P30-P52 NG2-Cre ERT2, TdTom, Lgl ${ }^{f l / f l}$ and NG2$C r e^{E R T 2}, T d T o m, ~ L g l 1^{w t / w t}$ mice were injected with tamoxifen twice daily for 4 consecutive days. FACS isolated OPC were subjected at P45 to microarray analyses. To assess proliferation and cell division modes in demyelinated lesions, demyelination was induced by a single LPC injection into the CC or the spinal cord of mice previously injected with tamoxifen. For differentiation, demyelination was induced by LPC injection after the last tamoxifen injection and mice were sacrificed 10 days after to evaluate the effect on OPC differentiation. For spinal cord white matter demyelination, mice were anesthetized with isofluorane and demyelination was induced by LPC into the ventral or dorsal white matter funiculus at the level of $\mathrm{T} 12^{68,69}$. For CC demyelination, LPC was injected in the right or the left hemisphere (AP+1 mm, $L \pm 1 \mathrm{~mm}, \mathrm{DV}-2.1 \mathrm{~mm})^{70}$. For EdU labeling, mice were injected i.p. with $10 \mathrm{mg} / \mathrm{mL}$ EdU twice a day, or EdU was mixed with drinking water at a low concentration $(0.1 \mathrm{mg} / \mathrm{mL})$ for 5-14 days. For long-term tracking of Lgl1 deletion effects, tamoxifen was injected at P30 for 5 consecutive days, and tissue analyzed by FACS at P120.

Cell culture. Cells were isolated from the CC, which includes the subcallosal zone of P30-P60 mice, and cultured as non-adherent spheres in proliferative medium with NT3, CNTF, PDGFA, and FGF2 (Peprotech) ${ }^{34}$. Differentiation was induced by plating cells on poly-L-Lysine coated glass-bottom 96-well plates (CORNING) at 5000 cells $/ \mathrm{mL}$ in medium containing CNTF, FGF2, and $\mathrm{T}^{2}$. Cells were incubated with inhibitor of endocytosis Pitstop1 at $25 \mu \mathrm{M}$. To induce Lgl1 deletion in vitro, cells were incubated with adenovirus-cre-GFP (Vector BioLabs) and adenovirus$\mathrm{GFP}^{2}$. Viability assay was performed using the Alamar Blue reagents ${ }^{71}$.

Pair assay. Cells were subjected to a pair assay by plating dissociated single cells at clonal density for $12-36 \mathrm{~h}$. Cell pairs formed were fixed and subjected to immunostaining with primary antibody and fluorescence-coupled secondary antibody ${ }^{2}$. Nuclei were visualized using DAPI (Sigma). Unless indicated otherwise, for pair assay a minimum of 50 and up to 200 cell pairs per cell type and condition, respectively, were analyzed, using a fluorescent microscope.

Immunofluorescence and in vivo cell pair assays. NG2-Cre $e^{E R T 2}, T d T o m, L g l 1^{f l / f l}$ and NG2-Cre $e^{E R T 2}$,TdTom, Lgl1 ${ }^{w t / w t}$ mice injected with tamoxifen were PFA perfused, brains were resected and frozen, sectioned and post-fixed for $10 \mathrm{~min}$ with $4 \%$ PFA, blocked with PBS 5\% normal goat serum (NGS) or normal donkey serum (NDS) and $0.1 \%$ Triton X-100 for $1 \mathrm{~h}$. For cell pair assays, sections were stained for anti-red fluorescence antibody (to detect the NG2+ cells and progeny with Lgl1 deletion) and anti-CC1 antibody overnight, washed and stained with fluorescence-couple secondary antibodies. Cell pairs were identified as two red fluorescence-positive (NG2Tom) and EdU-positive cells spaced less than $20 \mu \mathrm{m}^{2,3}$ The entire CC was counted as three or more distinct brain sections per animal in 4-5 individual animals per group. Cell pairs with one CC1-positive cell were scored as an ACD.

The following antibodies were used at the given concentration:

a-Rb NG2 (Millipore 1:200), aMs-IgM O4 (R\&D, 1:400), $\alpha$-Ms CC1 (Calbiochem, 1:100), a-Gt Dcx (Santa Cruz Biotechnology, 1:300), a-Gt Hugl-1 (Lgl1, Santa Cruz biotechnology, 1:200), a-Rb Hugl-1 (Lgl1, Santa Cruz Biotechnology, 1:200), $\alpha$-Ms Nestin (Chemicon international, 1:400), $\alpha$-Rb PlP (abcam105784, 1:1000), $\alpha$-Rat MBP (abcam7349, 1:400), $\alpha$-Rb-beta galactosidase (ab616, 1:200), gt- $\alpha$-Olig2 (EMD Millipore; 1:200), $\alpha$-Rb-Rab11 (Life Technologies Corporation, 1:200); $a-R b-p 19 A r f$ (Santa Cruz; 1:100); $\alpha$-Gt-Lamp1 (R and D systems AF4320, 1:100); $\alpha$-ARF 6 (Santa Cruz sc-7971; 1:200), a-Ms EEA1 (Santa Cruz sc-365652, 1:150); $\alpha$-Rb-Rab4 (Millipore 07-655; 1:200); $\alpha-M s-R a b 10$ (Life Technologies Corporation, 1:100).

For $\mathrm{O} 4$ staining, cells were stained live in vitro: $\mathrm{O} 4$ was incubated $1 \mathrm{~h}(1: 400)$ at $37^{\circ} \mathrm{C}$ in the corresponding culture medium (OPC proliferation or OL differentiation) before PFA fixation and further antibody staining.

EdU was visualized using the Click-it reaction kit (Life Technologies Corporation) following the manufacturer's instructions ${ }^{71}$

Early endocytosis compartment was visualized using the BacMam technology following manufacturer's instructions (Life Technologies Corporation). Immunofluorescent images were acquired on a Nikon Ti-E microscope with Nikon perfect focus system equipped with a Hamamatsu Flash4.0 camera equipped with a 40×/0.75 Plan Fluor objective and/or Nikon Ti Yokogawa CSU-22 spinning disk confocal equipped with a Plan Apo VC $60 \times / 1.4$ oil objective. Images were analyzed with NIS-Elements 4.2 (Build 982) and ImageJ.

FACS. NG2-Cre $e^{E R T 2}, T d T o m, L g l 1^{w t / w t}$ and, NG2-Cre ${ }^{E R T 2}$,TdTom, Lgl1 ${ }^{f l / f l}$ mice were analyzed by FACS 30 days or 120 days after tamoxifen injection at P30. Lateral ventricle walls and/or CC cells were isolated from Ascl1-GFP and NG2DsRed double-transgenic mice at P60. For DNA content analysis, dissociated cells were incubated with the vital DNA marker Hoechst 33342 (Sigma) ${ }^{72}$. The antibodies to identify distinct cell populations were anti-O4-biotin (MACS Miltenyi 
Biotec; 1:10) conjugated to streptavidin-FITC (MACS Miltenyi Biotec; 1:10); CD140a phycoerythrin-cyanine7 [PC7]-conjugated (mouse clone APA5; 1:100 eBiosciences); CD24 phycoerythrin-cyanine7 [PC7]-conjugated (Rat IgG2b; 1:100 Life Technologies); CD140b [APC]-conjugated (life technologies clone APB5, 1:50), and Glast-APC (MACS miltenyi, 1:30). Cells were gated following the fluorescence minus one (FMO) control. For microarray analyses, CC tissue was isolated from NG2-Cre ${ }^{E R T 2}$, TdTom, Lgll $l^{f l f l}(n=4)$ and NG2-Cre ${ }^{E R T 2}$, TdTom, $L g l 1^{w t / w t}(n=5)$ triple-transgenic, P30 mice injected twice daily with tamoxifen for 4 consecutive days and OPC were obtained by sorting for Tom-positive, PDGFRa-positive O4-negative cells.

To perform absolute cell counts, single cell suspensions were transferred to tubes containing a calibrated number of fluorescent beads (TruCount tubes, BD Biosciences) ${ }^{73}$. Prior to FACS sorting propidium iodide (PI) or Hoechst 33258 was added to a final concentration of $2 \mu \mathrm{g} / \mathrm{mL}$ to label the dead cells. Cells were analyzed and sorted on a FACS ARIA II (BD Biosciences). The data were analyzed with FlowJo data analysis software (Tree Star, Ashland, OR, USA).

Immunoblotting. Glioblastoma xenografts proteins were extracted using cell lysis buffer (Cell Signaling) supplemented with proteinase and phosSTOP phosphatase inhibitor cocktail (Roche). Proteins were separated by SDS-PAGE and transferred onto polyvinylidene difluoride membranes, which were then probed with primary antibodies, followed by horseradish peroxidase-conjugated secondary antibody, and visualized by ECL (GE Healthcare). Antibodies specific for Hugl1 and tubulin as loading control were from Sigma.

RNA extraction and reverse transcription. For all experiments, RNAs were extracted with QIAGEN RNeasy microkit and reverse transcribed into cDNAs using high capacity RNA to cDNA kit (Applied Biosystems).

Taqman low-density arrays. For the Taqman low-density array experiment, 200-500 cells/population were sorted from the CC of young adult Ascl1-GFP and NG2-DsRed double-transgenic mice. TaqMan Array Micro Fluidic Card were designed for neural stem cell, oligodendrocyte progenitor cell and differentiated oligodendrocyte markers, as well as well selected ACD regulators. A PreAmp pool was designed for these specific primers and a preamplification reaction was set up to 14 cycles according to manufacturer's instructions (Custom TaqMan ${ }^{\circ}$ PreAmp Pools Protocol, Applied Biosystems).

Whole-genome microarrays. For the microarray experiments 200-500 Lgll knockout and wildtype OPC cells (PDGFRa and red fluorescent protein positive O4 negative), were extracted with the RNA plus micro kit (QIAGEN) and reverse transcription into cDNAs was performed using the High Capacity Reverse Transcription Kit (Thermofisher scientific). Sample quality was controlled using the Agilent Bioanalyzer and samples were loaded on GeneChipR Mouse Gene 2.0 ST Arrays (Affymetrix). Microarray data were analyzed using Partek ${ }^{\oplus}$ Genomic Suite 6.6 with RMA normalization and differential gene expression detected with ANOVA. Ingenuity pathway analyses (QIAGEN bioinformatics) were used to identify targets and pathway differentially expressed between the Lgll WT and Lgll cKO samples.

Real-time quantitative reverse transcription PCR. Receptor-mediated endocytosis regulator genes were validated by qRT-PCR on OPC cultured from GFAPCre, $d T_{o m} f l f l$, Lgl wt $(n=6)$ vs. $L g l l^{f l f l}(n=6)$ mice using the following primers: Ap2al (5'-3': GGAACCTCCTGGTGGATGTC, 3'-5': AGGACCTGGGCTGAGGAAG); Epb4112 (5'-3': GCCCCAGTCAGGGAATTTCA, 3'-5': TTACCGCTTACACC GGACAC); Hiplr (5'-3': ATCTGGCCAGGAGCAGATTG, 3'-5': AAGACTCGC ACCTGTGTCTC) Eeal (5'-3': TCTTCAGAGGGTTTCATATGTCCC, $3^{\prime}-5^{\prime}$ : TG AGGCCTGTAGGTCTTGGA) Reps1 (5'-3': GCAGATACTCCACCAACCAGT, 3'-5': GTACTGTCGTCTGGTCCTGG); Syne1 (5'-3': GACATGCAGGGAGTCA CACA, 3'-5': CTTTTTGCTGCTGGAGGCTG); Pld2 (5'-3': ATCCT-

GAAGGCTCACGAACAG, 3'-5': GAAGAAAGGGTGAAGGAGGCA); p19Arf (Forward: 5'-3': TGAGGCTAGAGAGGATCTTGAGAAG, Reverse: 5' $-3^{\prime}$ : GTGAACGTTGCCCATCATCATC); LLgl1 (exon 2-3 Forward: 5'-3': CCACAA GACTGTGGAGCATGG, Reverse: 5'-3': AAA-TGC-ATC-TGG-GTG-ACG-GT).

Transferrin uptake. The CC of GFAP-Cre, $d T^{\text {Tom }}{ }^{l o x} / l o x, L g l 1{ }^{w t / w t}(n=4)$ vs. $L g l 1$ ${ }^{o x} / l o x(n=4)$ was extracted from young adult mice (P60). After enzymatic digestion with papain $(10 \mathrm{~min}, 15 \mathrm{U} / \mathrm{mL})^{72}$, cells were washed with cold wash solution (PBS containing $20 \mathrm{mM}$ glucose and 1\% BSA). Then Transferrin Alexa Fluor 488 conjugate (Molecular probes, T13342) ${ }^{74}$ was added at $25 \mu \mathrm{g} / \mathrm{mL}$ and incubated at $37^{\circ} \mathrm{C}$ for $15 \mathrm{~min}$. Non-internalized ligand was removed from the cell surface using three 5 min wash with ice-cold freshly prepared stripping buffer (PBS/0.15\% BSA, pH 3.5 adjusted with HCL) and washed three times with cold wash solution. Data analysis to quantify changes in Transferrin fluorescence in dTom + cells was performed using a FACS Aria II (BD Biosciences) and analyzed with FlowJo data analysis software (Tree Star, Ashland, OR, USA).
Live staining of cells. Plated $L g l l \mathrm{cKO}$ and WT OPC were incubated on ice at $4{ }^{\circ} \mathrm{C}$ for $2 \mathrm{~h}$ with $\alpha$-NG2-EC (Rabbit, courtesy of Dr. Stallcup) in Neurobasal medium (3\% BSA). After two washes with neurobasal medium, cells were incubated for $1 \mathrm{~h}$ with an Alexa488 goat $\alpha$-Rabbit. For lysosome tracking, cultured OPC were incubated with CellLight Reagent BacMam 2.0 (Molecular probe) $24 \mathrm{~h}$ prior to live imaging targeting the early endosome Rab5a (C10586) and lysosome-specific protein Lamp1 (C10507) at a concentration of 50 PPC.

Fluorescence time lapse microscopy. Time lapse movies were taken under a conventional widefield epifluorescence system (Nikon) equipped with a $37^{\circ} \mathrm{C}$ incubation chamber and $\mathrm{CO}_{2}$ control for live cell imaging. Live-labeled cells were incubated in differentiation conditions and the system programed to take fluorescent pictures at the following frequencies: 1 frame $/ 30 \mathrm{~s}$ to follow early NG2 endocytosis and 1 frame/ $1 \mathrm{~h}$ to follow its trafficking towards lysosomes.

TIRF microscopy. Live labeled cells (NG2-EC) were plated in differentiation conditions and placed on a coupled incubator for live cell imaging $\left(37^{\circ} \mathrm{C}, 5 \% \mathrm{CO}_{2}\right)$ under the TIRF microscope. Fluorescent pictures were taken at different frequencies: 1 frame $/ 30$ min or 1 frame/30 s. TIRF was used to provide a mean to selectively excite fluorophore very near the solid surface $(<100 \mathrm{~nm})$ without exciting fluorescence from regions farther from the surface. Therefore, any Alexa488 positive dot in perfect focus was considered as a docking NG2 on the membrane region that is being imaged. Different quantities of NG2 recycling over time were represented as total corrected fluorescence measured using ImageJ.

Recycling inhibition. Lgll cKO OPC were treated with differentiation medium containing $10 \mu \mathrm{M}$ Monensin (TOCRIS, 5223) for $4 \mathrm{~h}$. Cells were monitored under TIRF for NG2 recycling monitoring during the first hour of treatment or were switched back to normal differentiation medium for 5 days before being fixed and immunostained for O4 and NG2. Endocytosis of the proteoglycan NG2 was followed using a mouse-anti-NG2.EC antibody against the extracellular domain of NG2 (courtesy of Dr. Stallcup). OPC cultures were incubated $24 \mathrm{~h}$ prior to live imaging with CellLight ${ }^{\circledR}$ Reagents BacMam 2.0 (Molecular Probes) targeting the early endosome-specific protein Rab5a (ref C10586) of lysosome-specific protein Lamp1 (ref C10507) at a concentration of 50 PPC. Cells were starved $2 \mathrm{~h}$ prior to imaging in NBA medium, and $50 \mathrm{ng} / \mathrm{mL}$ of PDGF-AA was added right before imaging to induce endocytosis. Time-lapse movies were taken over time spans of 30-45 min with $30 \mathrm{~s}$ interval between the images to follow early endocytosis of NG2 antibody and 2-3 h with a 30 min interval to follow its internalization to the lysosome. After live imaging, cells were fixed $10 \mathrm{~min}$ in PFA4\%, stained with the appropriate antibodies and imaged with a Nikon Ti Yokogawa CSU-22 spinning disk confocal equipped with a Plan Apo VC 60×/1.4 oil objective.

Endocytosis inhibitor. Monensin (TOCRIS, 5223), a selective recycling endocytosis $^{75}$, was used and was not toxic at a working concentration of $10 \mu \mathrm{M}$.

Univariate Cox model. The prognostic significance of high Lgll expression ( $\geq$ median) on overall survival (defined as the time between diagnosis and death due to any cause) was retrospectively tested. The follow-up period ranged from 0 to 8 years (median $=1$ year). The number of events that occurred during this period and the number of censored individuals are: 86 events, with 13 censored.

Statistics and analysis. Statistical analyses were conducted as indicated in the figure legends using GraphPad Prism 5.0 and Microsoft Excel. A Mann and Whitney two-tailed paired $t$-test was conducted to analyze for significant differences between two treatment groups. For Kaplan-Meier survival rate analyses, the number of surviving mice were recorded daily. The data were subjected to Logrank test in order to determine if significant differences existed in survival between the Lgll knockout and control groups. For statistical tests ${ }^{*} p<0.05,{ }^{* *} p<0.01$, ${ }^{* * *} p$ $<0.001$. A univariate Cox models was fitted on the survival data of the 99 glioma patients (92 GBM and $7 \mathrm{LGG}$ ) to obtain p-values. R-function 'survfit' (package 'survival') was used to generate survival curves from the previously fitted Cox model.

Data availability. The data that support the findings of this study are available from the corresponding author upon reasonable request.

Received: 26 October 2017 Accepted: 13 June 2018

Published online: 21 August 2018

\section{References}

1. Young, K. M. et al. Oligodendrocyte dynamics in the healthy adult CNS: evidence for myelin remodeling. Neuron 77, 873-885 (2013). 
2. Sugiarto, S. et al. Asymmetry-defective oligodendrocyte progenitors are glioma precursors. Cancer Cell 20, 328-340 (2011).

3. Boda, E. et al. Early phenotypic asymmetry of sister oligodendrocyte progenitor cells after mitosis and its modulation by aging and extrinsic factors. Glia 63, 271-286 (2015).

4. Nishiyama, A., Lin, X. H., Giese, N., Heldin, C. H. \& Stallcup, W. B. Interaction between NG2 proteoglycan and PDGF alpha-receptor on O2A progenitor cells is required for optimal response to PDGF. J. Neurosci. Res. 43, 315-330 (1996).

5. Goretzki, L., Burg, M. A., Grako, K. A. \& Stallcup, W. B. High-affinity binding of basic fibroblast growth factor and platelet-derived growth factor-AA to the core protein of the NG2 proteoglycan. J. Biol. Chem. 274, 16831-16837 (1999).

6. Noble, M., Murray, K., Stroobant, P., Waterfield, M. D. \& Riddle, P. Plateletderived growth factor promotes division and motility and inhibits premature differentiation of the oligodendrocyte/type- 2 astrocyte progenitor cell. Nature 333, 560-562 (1988).

7. Kucharova, K. \& Stallcup, W. B. The NG2 proteoglycan promotes oligodendrocyte progenitor proliferation and developmental myelination. Neuroscience 166, 185-194 (2010).

8. Bin, J. M., Harris, S. N. \& Kennedy, T. E. The oligodendrocyte-specific antibody 'CC1' binds Quaking 7. J. Neurochem. 139, 181-186 (2016).

9. Zhu, X. et al. Age-dependent fate and lineage restriction of single NG2 cells. Development 138, 745-753 (2011).

10. Hill, R. A., Patel, K. D., Goncalves, C. M., Grutzendler, J. \& Nishiyama, A. Modulation of oligodendrocyte generation during a critical temporal window after NG2 cell division. Nat. Neurosci. 17, 1518-1527 (2014).

11. Assanah, M. et al. Glial progenitors in adult white matter are driven to form malignant gliomas by platelet-derived growth factor-expressing retroviruses. J. Neurosci. 26, 6781-6790 (2006).

12. Lindberg, N., Kastemar, M., Olofsson, T., Smits, A. \& Uhrbom, L. Oligodendrocyte progenitor cells can act as cell of origin for experimental glioma. Oncogene 28, 2266-2275 (2009).

13. Persson, A. I. et al. Non-stem cell origin for oligodendroglioma. Cancer Cell 18, 669-682 (2010)

14. Liu, C. et al. Mosaic analysis with double markers reveals tumor cell of origin in glioma. Cell 146, 209-221 (2011).

15. Feutlinske, F. et al. Stonin1 mediates endocytosis of the proteoglycan NG2 and regulates focal adhesion dynamics and cell motility. Nat. Commun. 6, 8535 (2015).

16. McMahon, H. T. \& Boucrot, E. Molecular mechanism and physiological functions of clathrin-mediated endocytosis. Nat. Rev. Mol. Cell Biol. 12, 517-533 (2011).

17. Gateff, E. \& Mechler, B. M. Tumor-suppressor genes of Drosophila melanogaster. Crit. Rev. Oncog. 1, 221-245 (1989).

18. Peng, C. Y., Manning, L., Albertson, R. \& Doe, C. Q. The tumour-suppressor genes $\operatorname{lgl}$ and dlg regulate basal protein targeting in Drosophila neuroblasts. Nature 408, 596-600 (2000).

19. Vasioukhin, V. Lethal giant puzzle of Lgl. Dev. Neurosci. 28, 13-24 (2006).

20. Sripathy, S., Lee, M. \& Vasioukhin, V. Mammalian Llgl2 is necessary for proper branching morphogenesis during placental development. Mol. Cell Biol. 31, 2920-2933 (2011).

21. Klezovitch, O., Fernandez, T. E., Tapscott, S. J. \& Vasioukhin, V. Loss of cell polarity causes severe brain dysplasia in Lgl1 knockout mice. Genes Dev. 18, 559-571 (2004).

22. Jossin, Y. et al. Llgll connects cell polarity with cell-cell adhesion in embryonic neural stem cells. Dev. Cell 41, 481-495 e485 (2017).

23. Beattie, R. et al. Mosaic analysis with double markers reveals distinct sequential functions of Lgl1 in neural stem cells. Neuron 94, 517-533 e513 (2017).

24. Ozawa, T. et al. Most human non-GCIMP glioblastoma subtypes evolve from a common proneural-like precursor glioma. Cancer Cell 26, 288-300 (2014).

25. Gont, A. et al. PTEN loss represses glioblastoma tumor initiating cell differentiation via inactivation of Lgl1. Oncotarget 4, 1266-1279 (2013).

26. Gont, A. et al. Inhibition of glioblastoma malignancy by Lgl1. Oncotarget $\mathbf{5}$, 11541-11551 (2014).

27. Zhang, Y. et al. An RNA-sequencing transcriptome and splicing database of glia, neurons, and vascular cells of the cerebral cortex. J. Neurosci. 34, 11929-11947 (2014).

28. Hill, R. A. \& Nishiyama, A. NG2 cells (polydendrocytes): listeners to the neural network with diverse properties. Glia 62, 1195-1210 (2014).

29. Madisen, L. et al. A robust and high-throughput Cre reporting and characterization system for the whole mouse brain. Nat. Neurosci. 13, 133-140 (2010).

30. Kang, S. H., Fukaya, M., Yang, J. K., Rothstein, J. D. \& Bergles, D. E. NG2 $+\mathrm{CNS}$ glial progenitors remain committed to the oligodendrocyte lineage in postnatal life and following neurodegeneration. Neuron 68, 668-681 (2010).
31. Daynac, M. \& Petritsch, C. K. Regulation of asymmetric cell division in mammalian neural stem and cancer precursor cells. Results Probl. Cell Differ. 61, 375-399 (2017)

32. Franklin, R. J. \& Ffrench-Constant, C. Remyelination in the CNS: from biology to therapy. Nat. Rev. Neurosci. 9, 839-855 (2008).

33. Reynolds, R. et al. The response of NG2-expressing oligodendrocyte progenitors to demyelination in MOG-EAE and MS. J. Neurocytol. 31, 523-536 (2002).

34. Dugas, J. C., Tai, Y. C., Speed, T. P., Ngai, J. \& Barres, B. A. Functional genomic analysis of oligodendrocyte differentiation. J. Neurosci. 26, 10967-10983 (2006).

35. Calzolari, F. et al. Fast clonal expansion and limited neural stem cell selfrenewal in the adult subependymal zone. Nat. Neurosci. 18, 490-492 (2015).

36. Lindberg, N. et al. Oncogenic signaling is dominant to cell of origin and dictates astrocytic or oligodendroglial tumor development from oligodendrocyte precursor cells. J. Neurosci. 34, 14644-14651 (2014).

37. Zhu, X., Bergles, D. E. \& Nishiyama, A. NG2 cells generate both oligodendrocytes and gray matter astrocytes. Development 135, 145-157 (2008).

38. Huillard, E. et al. Cooperative interactions of BRAFV600E kinase and $\mathrm{CDKN} 2 \mathrm{~A}$ locus deficiency in pediatric malignant astrocytoma as a basis for rational therapy. Proc. Natl Acad. Sci. USA 109, 8710-8715 (2012).

39. Lerner, R. G. et al. Targeting a Plk1-controlled polarity checkpoint in therapyresistant glioblastoma-propagating cells. Cancer Res. 75, 5355-5366 (2015).

40. Verhaak, R. G. et al. Integrated genomic analysis identifies clinically relevant subtypes of glioblastoma characterized by abnormalities in PDGFRA, IDH1, EGFR, and NF1. Cancer Cell 17, 98-110 (2010).

41. Nishiyama, A., Dahlin, K. J., Prince, J. T., Johnstone, S. R. \& Stallcup, W. B. The primary structure of NG2, a novel membrane-spanning proteoglycan. $J$. Cell Biol. 114, 359-371 (1991).

42. Wang, T. et al. Lgl1 activation of Rab10 promotes axonal membrane trafficking underlying neuronal polarization. Dev. Cell 21, 431-444 (2011).

43. Stein, B. S., Bensch, K. G. \& Sussman, H. H. Complete inhibition of transferrin recycling by monensin in K562 cells. J. Biol. Chem. 259, 14762-14772 (1984)

44. He, L. \& Lu, Q. R. Coordinated control of oligodendrocyte development by extrinsic and intrinsic signaling cues. Neurosci. Bull. 29, 129-143 (2013).

45. Sakry, D., Karram, K. \& Trotter, J. Synapses between NG2 glia and neurons. J. Anat. 219, 2-7 (2011)

46. Gomez-Lopez, S., Lerner, R. G. \& Petritsch, C. Asymmetric cell division of stem and progenitor cells during homeostasis and cancer. Cell Mol. Life Sci. 71, 575-597 (2014)

47. Dimou, L. \& Gallo, V. NG2-glia and their functions in the central nervous system. Glia 63, 1429-1451 (2015).

48. Ge, W. P., Zhou, W., Luo, Q., Jan, L. Y. \& Jan, Y. N. Dividing glial cells maintain differentiated properties including complex morphology and functional synapses. Proc. Natl Acad. Sci. USA 106, 328-333 (2009).

49. Kukley, M., Capetillo-Zarate, E. \& Dietrich, D. Vesicular glutamate release from axons in white matter. Nat. Neurosci. 10, 311-320 (2007).

50. Hughes, E. G., Kang, S. H., Fukaya, M. \& Bergles, D. E. Oligodendrocyte progenitors balance growth with self-repulsion to achieve homeostasis in the adult brain. Nat. Neurosci. 16, 668-676 (2013).

51. Franklin, R. J. \& Goldman, S. A. Glia disease and repair-remyelination. Cold Spring Harb. Perspect. Biol. 7, a020594 (2015).

52. Tep, C. et al. Brain-derived neurotrophic factor (BDNF) induces polarized signaling of small GTPase (Rac1) protein at the onset of Schwann cell myelination through partitioning-defective 3 (Par3) protein. J. Biol. Chem. 287, 1600-1608 (2012).

53. Galvao, R. P. et al. Transformation of quiescent adult oligodendrocyte precursor cells into malignant glioma through a multistep reactivation process. Proc. Natl Acad. Sci. USA 111, E4214-E4223 (2014).

54. Shivas, J. M., Morrison, H. A., Bilder, D. \& Skop, A. R. Polarity and endocytosis: reciprocal regulation. Trends Cell Biol. 20, 445-452 (2010).

55. Holland, E. C., Hively, W. P., Gallo, V. \& Varmus, H. E. Modeling mutations in the G1 arrest pathway in human gliomas: overexpression of CDK4 but not loss of INK4a-ARF induces hyperploidy in cultured mouse astrocytes. Genes Dev. 12, 3644-3649 (1998).

56. Ozawa, T. et al. PDGFRA gene rearrangements are frequent genetic events in PDGFRA-amplified glioblastomas. Genes Dev. 24, 2205-2218 (2010)

57. Stallcup W. B. NG2 proteoglycan enhances brain tumor progression by promoting beta- 1 integrin activation in both cis and trans orientations. Cancers 9, 31 (2017).

58. de Vreede, G. et al. The Scribble module regulates retromer-dependent endocytic trafficking during epithelial polarization. Development 141, 2796-2802 (2014).

59. Chandrasekar, I. et al. Nonmuscle myosin II is a critical regulator of clathrinmediated endocytosis. Traffic 15, 418-432 (2014).

60. Wang, H. et al. Myosin II is a negative regulator of oligodendrocyte morphological differentiation. J. Neurosci. Res. 90, 1547-1556 (2012). 
61. Dahan, I., Yearim, A., Touboul, Y. \& Ravid, S. The tumor suppressor Lgl1 regulates NMII-A cellular distribution and focal adhesion morphology to optimize cell migration. Mol. Biol. Cell 23, 591-601 (2012).

62. Stanley, A. C. et al. The Rho GTPase Racl is required for recycling endosomemediated secretion of TNF in macrophages. Immunol. Cell Biol. 92, 275-286 (2014).

63. Tanaka, Y. et al. The phospholipid flippase ATP9A is required for the recycling pathway from the endosomes to the plasma membrane. Mol. Biol. Cell 27, 3883-3893 (2016).

64. Parsons, L. M., Portela, M., Grzeschik, N. A. \& Richardson, H. E. Lgl regulates Notch signaling via endocytosis, independently of the apical aPKC-Par6-Baz polarity complex. Curr. Biol. 24, 2073-2084 (2014).

65. Portela, M., Parsons, L. M., Grzeschik, N. A. \& Richardson, H. E. Regulation of Notch signaling and endocytosis by the Lgl neoplastic tumor suppressor. Cell Cycle 14, 1496-1506 (2015).

66. Zhuo, L. et al. hGFAP-cre transgenic mice for manipulation of glial and neuronal function in vivo. Genesis 31, 85-94 (2001).

67. Gong, S. et al. A gene expression atlas of the central nervous system based on bacterial artificial chromosomes. Nature 425, 917-925 (2003).

68. Arnett, H. A. et al. bHLH transcription factor Olig1 is required to repair demyelinated lesions in the CNS. Science 306, 2111-2115 (2004).

69. Zawadzka, M. et al. CNS-resident glial progenitor/stem cells produce Schwann cells as well as oligodendrocytes during repair of CNS demyelination. Cell Stem Cell 6, 578-590 (2010).

70. Rafalski, V. A. et al. Expansion of oligodendrocyte progenitor cells following SIRT1 inactivation in the adult brain. Nat. Cell Biol. 15, 614-624 (2013).

71. Grossauer, S. et al. Concurrent MEK targeted therapy prevents MAPK pathway reactivation during BRAFV600E targeted inhibition in a novel syngeneic murine glioma model. Oncotarget 7, 75839-75853 (2016).

72. Daynac, M. et al. Quiescent neural stem cells exit dormancy upon alteration of GABAAR signaling following radiation damage. Stem Cell Res. 11, 516-528 (2013).

73. Daynac, M. et al. TGFbeta lengthens the G1 phase of stem cells in aged mouse brain. Stem Cells 32, 3257-3265 (2014).

74. Tacheva-Grigorova, S. K., Santos, A. J., Boucrot, E. \& Kirchhausen, T. Clathrin-mediated endocytosis persists during unperturbed mitosis. Cell Rep. 4, 659-668 (2013).

75. Kaiser, J., Stockert, R. J. \& Wolkoff, A. W. Effect of monensin on receptor recycling during continuous endocytosis of asialoorosomucoid. Exp. Cell Res 174, 472-480 (1988)

\section{Acknowledgements}

We thank Banafsheh Kadkhodaei, Ian D. Meyers, Helena Oft, Sonia Spindt and Jacky Huang for technical assistance and Valeri Vasioukhin for resources. We would like to acknowledge DeLaine Larsen and Kari Herrington at the Nikon Imaging Center at UCSF for technical support. We thank Martin Oft, Amy Sprowles, Russ Pieper, and William A Weiss for their critical comments. S.F. is a Harry Weaver Neuroscience Scholar of the National Multiple Sclerosis Society. Funding was provided by the National Cancer Institute (R01CA16476-04 to C.K.P.) and the National Institute of Neurological Disorders and Stroke (R01NS080619 to C.K.P.; R01NS097551 to S.F.) of the National Institute of Health; the Loglio Research Program (to C.K.P.), the Research Allocation Program Investigator Grant UCSF (to C.K.P.), the University of California Cancer Research Coordination Committee (CA-0095729 to C.K.P.), and California Institute of Regenerative Medicine (to H.Y.C).

\section{Author contributions}

Conceptualization, M.D., M.C., and C.K.P.; Methodology, M.D., M.C., S.F., C.K.P. Investigation, M.D., M.C., H.Y.C.; N.E.M., N.A., J.N.; Resources, W.S.; Writing-original draft, M.D., C.K.P.; Writing-review and editing, M.D., M.C., and C.K.P.; Funding acquisition, C.K.P.; Supervision, C.K.P.

\section{Additional information}

Supplementary Information accompanies this paper at https://doi.org/10.1038/s41467018-05099-3.

Competing interests: The authors declare no competing interests.

Reprints and permission information is available online at http://npg.nature.com/ reprintsandpermissions/

Publisher's note: Springer Nature remains neutral with regard to jurisdictional claims in published maps and institutional affiliations.

Open Access This article is licensed under a Creative Commons Attribution 4.0 International License, which permits use, sharing, adaptation, distribution and reproduction in any medium or format, as long as you give appropriate credit to the original author(s) and the source, provide a link to the Creative Commons license, and indicate if changes were made. The images or other third party material in this article are included in the article's Creative Commons license, unless indicated otherwise in a credit line to the material. If material is not included in the article's Creative Commons license and your intended use is not permitted by statutory regulation or exceeds the permitted use, you will need to obtain permission directly from the copyright holder. To view a copy of this license, visit http://creativecommons.org/ licenses/by/4.0/.

(c) The Author(s) 2018 